\title{
Accurate Symbolic Solution of Ginzburg-Landau Equations in the Circular Cell Approximation by Variational Method: Magnetization of Ideal Type II Superconductor
}

\author{
0. A. Chevtchenko \\ HTS-powercables.nl BV, Apeldoorn, The Netherlands \\ Email: o.a.chevtchenko@gmail.com
}

How to cite this paper: Chevtchenko, O.A. (2017) Accurate Symbolic Solution of Ginzburg-Landau Equations in the Circular Cell Approximation by Variational Method: Magnetization of Ideal Type II Superconductor. Journal of Modern Physics, 8, 982-1011.

https://doi.org/10.4236/jmp.2017.86062

Received: April 30, 2017

Accepted: May 28, 2017

Published: May 31, 2017

Copyright $\odot 2017$ by author and Scientific Research Publishing Inc. This work is licensed under the Creative Commons Attribution International License (CC BY 4.0).

http://creativecommons.org/licenses/by/4.0/

\begin{abstract}
In this paper I access the degree of approximation of known symbolic approach to solving of Ginzburg-Landau (GL) equations using variational method and a concept of vortex lattice with circular unit cells, refine it in a clear and concise way, identify and eliminate the errors. Also, I will improve its accuracy by providing for the first time precise dependencies of the variational parameters; correct and calculate magnetisation, compare it with the one calculated numerically and conclude they agree within $98.5 \%$ or better for any value of the GL parameter $k$ and at magnetic field $0.01 \leq \bar{B} / B_{c 2} \leq 1$, which is good basis for many engineering applications. As a result, a theoretical tool is developed using known symbolic solutions of GL equations with accuracy surpassing that of any other known symbolic solution and approaching that of numerical one.
\end{abstract}

\section{Keywords}

Ginzburg-Landau Equations, Accurate Symbolic Solution, Circular Unit Cell

\section{Introduction}

Much of basic superconductor behavior in magnetic field can be understood from the phenomenological model expressed by two Ginzburg-Landau (GL) equations [1] [2]. Known numerical methods produce excellent and reliable results along with "...difficulty of a numerical solution of the complex-valued GL equations" [3] [4], the demands of calculation effort and time and being less transparent. Calculated magnetisation curves for vortex lattices with rectangular, hexagonal or circular unit cells coincide within line thickness [3]. This fact mo- 
tivates solving GL equations symbolically that currently lags behind due to the complexity of the problem [5]-[10]. The lower critical field $B_{c 1}$ can be accurately calculated symbolically, see Section 6.1. In strong magnetic fields the symbolic approximation [7] holds, see Section 4.2. At medium magnetic fields a symbolic approximation considering vortex lattice of circular unit cells is developed [5] [6] [8] [10]. The drawbacks of [5] [6] are dealt with in [8] [9] [10] and will not be discussed here. As stated in [8] [9] [10], their method approximately describes magnetic properties of type II superconductors with periodic lattice over the entire range of magnetic fields and for any value of the GL parameter $k=\lambda / \xi \geq k_{12}=2^{-1 / 2}$ with $\lambda, \xi$ London penetration depth and the coherence length respectively. Moreover, the magnetic field dependence of (reversible) magnetisation can be calculated in seconds.

However, a simple check shows that magnetisation calculated with this method ([8] [9] [10], as published) when compared to that calculated numerically [3], has relative differences exceeding 25\%, which raises concerns about the accuracy. Clearly, the accuracy of the obtained solution of GL equations is vital: a symbolic one only has added value when its accuracy is comparable to that of the numerics. In this paper I will access the degree of approximation of the method [8] [9] [10], refine it in a clear, concise and rational way, identify and eliminate the errors. Furthermore, I will improve accuracy of the method by providing for the first time accurate dependencies of the variational parameters; correct and calculate magnetisation, compare it with the one calculated numerically and conclude they agree for any $k$ within $98.5 \%$ or better at magnetic field $0.01 \leq \bar{B} / B_{c 2} \leq 1$, which is sufficient for many engineering applications.

\section{Theoretical Formalism}

\subsection{Normalisation}

In this paper I omit the time-dependent terms in the GL equations written in SI units [11] [12] and use almost the same normalisation as in [3] [11] [12], except that I normalise all length-related quantities by the penetration depth $\lambda=k \xi$ so that both $B_{c 2}$ and $\lambda$ are defined through $\xi$ and Annex provides more details. Below there are six mean (averaged over the unit cell area) magnetic field magnitudes (magnetic flux densities) in use: thermodynamic (equilibrium, applied) field $B_{a}$, magnetisation $M$ (from the induced currents), the resulting (total) field: $b_{m}=\bar{b}=\int_{0}^{R} b_{r} \frac{2 \pi r d r}{\pi R^{2}}$ with the local field $b_{r}$ defined by GL equations in Table 1; the lower, the upper and thermodynamic critical magnetic fields: $B_{c 1}, B_{c 2}$ and $k B_{c} \sqrt{2}=B_{c 2}$ respectively. Furthermore, in this paper $B_{c 2}=\frac{\Phi_{0}}{2 \pi \xi^{2}}$ is considered independent of $k$ or $B$, defined externally (through $\xi$ and BCS theory [2]) and at the end used as a scaling factor for all magnetic fields; $\Phi_{0}$ is magnetic flux quantum. Note the local magnetic flux density $b_{z k}(r) / k=B_{z}(r) / B_{c 2}=$ $b_{r}$ introduced (along with the four other local quantities) in Table 1 and Section 2.4. Here and below I use the dimensionless units: distance $r$, modulus of the magnetic vector potential $a_{\varphi}(r)$, modulus of the super-velocity $q_{\varphi}(r)$, 
Table 1. Two GL equations written in forms 1, 2 for a unit cell with axial symmetry.

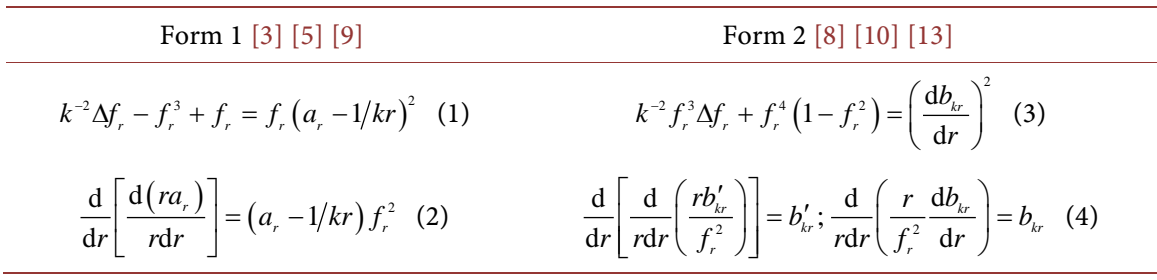

magnetic induction $\bar{b}=b_{m}=\bar{B} / B_{c 2}$ and $\quad \bar{b}_{k}=\frac{\bar{B}}{\sqrt{2} B_{c}}=\bar{b} k$, current density $j_{\varphi}(r)$, magnetic flux quantum $\frac{\Phi_{0}}{k^{2} \xi^{2} \sqrt{2} B_{c}}=\frac{2 \pi}{k}$, the free energy density $F$ and the magnitude $f_{r}=f(r)=|\psi|$ of the complex GL order parameter $\psi$ are scaled by the dimensioned units: $\lambda=\xi k, \xi B_{c} \sqrt{2}, \xi B_{c} \sqrt{2}, B_{c 2}=\mu_{o} H_{c 2}$ and $\sqrt{2} B_{c}, \frac{H_{c} \sqrt{2}}{\lambda}, \lambda^{2} \mu_{0} H_{c} \sqrt{2}, F_{c}=\mu_{0} 2 H_{c}^{2}$ the energy density ( $\mu_{0}$ is magnetic permeability of vacuum) and by the GL coefficients $\sqrt{-\alpha / \beta}=\left|\psi_{0}\right|$ respectively, see Table A5 for more. All dimensioned units use the International System of Units SI.

\subsection{Variational Method}

The variational method [5] [6] [8] [9] [10] assumes for the dimensionless modulus $|\psi|$ of the complex-valued order parameter $\psi=f(r) \exp (-i k \vartheta)$ a trial function $f_{r}=f_{r}(r)$ :

$$
|\psi|^{2}=f_{r}^{2}=\left(r f_{\infty} / \rho_{r}\right)^{2},
$$

where the notation $\rho_{r}^{2}=r^{2}+\xi_{v}^{2}$ is introduced; the $f_{\infty} \geq 0, \quad \xi_{v} \neq 0$ are two variational parameters representing respectively the depression of the order parameter due to overlapping vortices and the effective core radius of a vortex; $r, \varphi$ are the radial coordinate of cylindrical coordinate system and the phase angle respectively (a vortex line is centered on the $\mathrm{z}$-axis, so that $r^{2}=x^{2}+y^{2}$; $\varphi=\arctan (y / x) ; x, y, z$ are rectangular system coordinates, etc. [8]). Both variational parameters $f_{\infty}$ and $\xi_{v}$ depend only on magnetic field $\bar{b}$ and on the GL parameter $k$ (Figures 1-4), so that e.g., $\rho_{r} \mathrm{~d} \rho_{r}=r \mathrm{~d} r$. The order parameter $\psi$ is interpreted as the local density of the Cooper pairs and its phase $\vartheta$ is determined by the electric potential [12].

\subsection{Free Energy Density of Superconductor}

Averaged over the cell area Helmholtz dimensionless free energy density $F$ of a circular unit cell (with radius $R$ ) has two contributions [3] [8] [9] [10]:

$$
\begin{gathered}
F=F_{\text {em }}+F_{\text {core }}, \\
F_{\text {em }}=\int_{0}^{R}\left[f_{r}^{2} q_{r}^{2}+b_{k r}^{2}\right] \frac{2 \pi r \mathrm{~d} r}{\pi R^{2}}, \\
F_{\text {core }}=\int_{0}^{R}\left[\frac{1}{2}\left(1-f_{r}^{2}\right)^{2}+\frac{\left(f_{r}^{\prime}\right)^{2}}{k^{2}}\right] \frac{2 \pi r d r}{\pi R^{2}},
\end{gathered}
$$




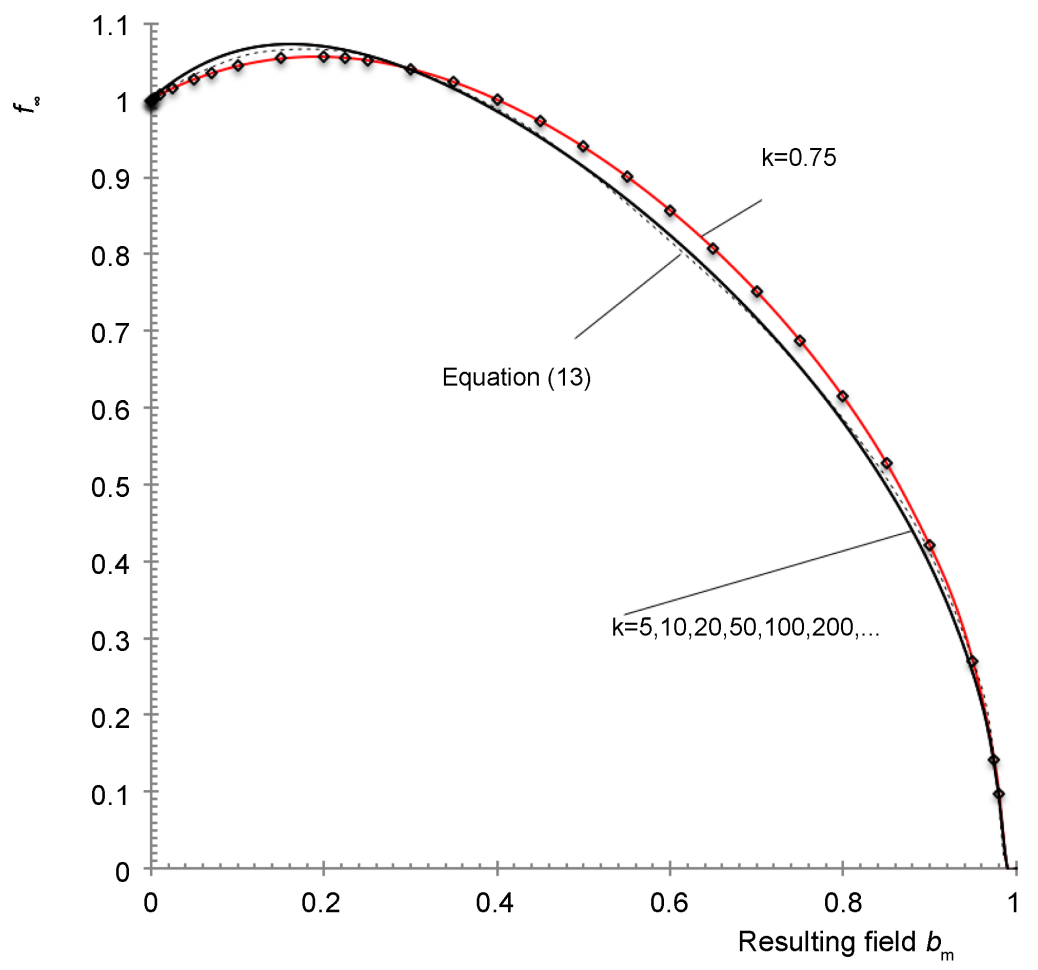

Figure 1. Calculated variational parameter $f_{\infty}$ vs. magnetic field $b_{m}$, all dots from Annex for $k \geq 5$ collapse at one curve approximated by the splines (visible here as the solid black line marked " $k \geq 5$ ", the dashed black line is from Equation (18) and the solid red line is the cubic spline fit at $k=0.75$, indicating the range of the deviation from the curve $k \geq 5$ ).

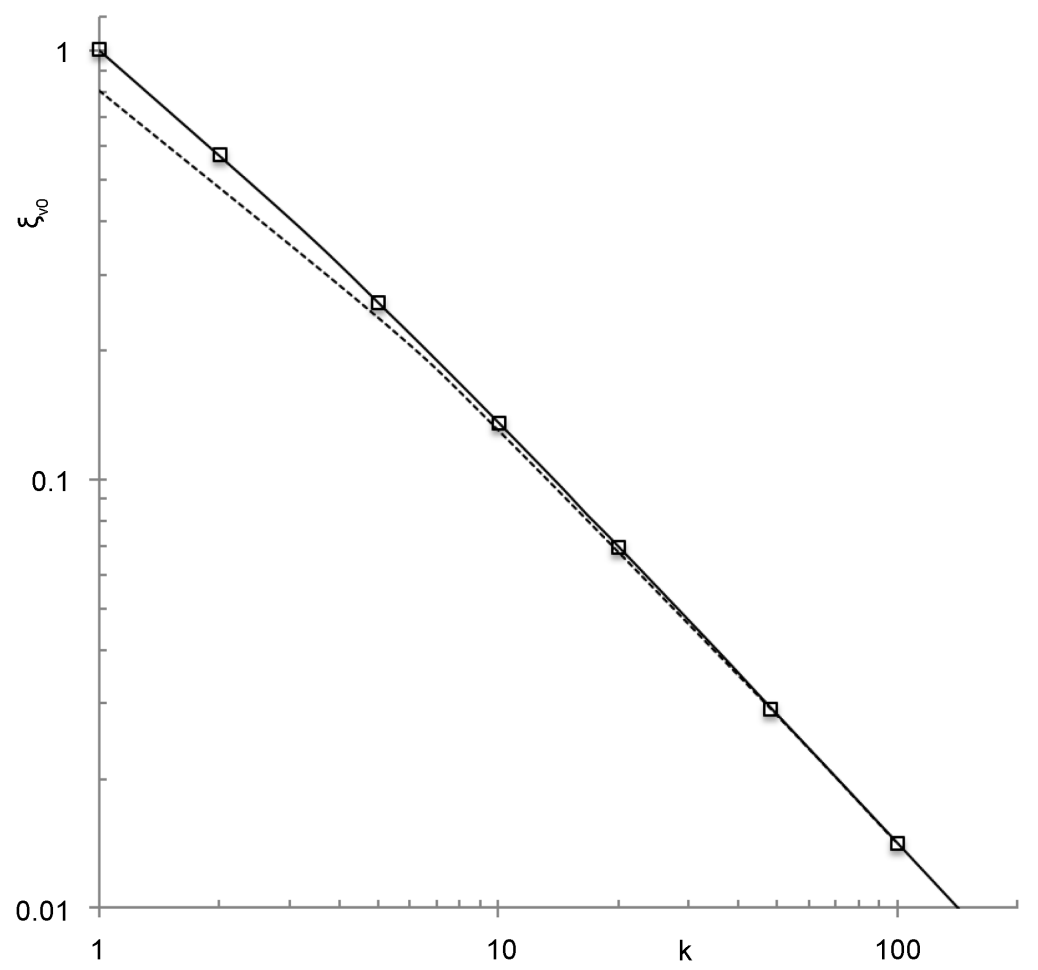

Figure 2. Calculated dependence of the variational parameter $\xi_{v 0}$ on $k$ at $\bar{b} \rightarrow 0:$ the boxes represent the data from Annex (the box size corresponds to the relative error of 7\%); the solid line-[[5], Equation (16)], the dashed line-[[8], Equation (15)]. 


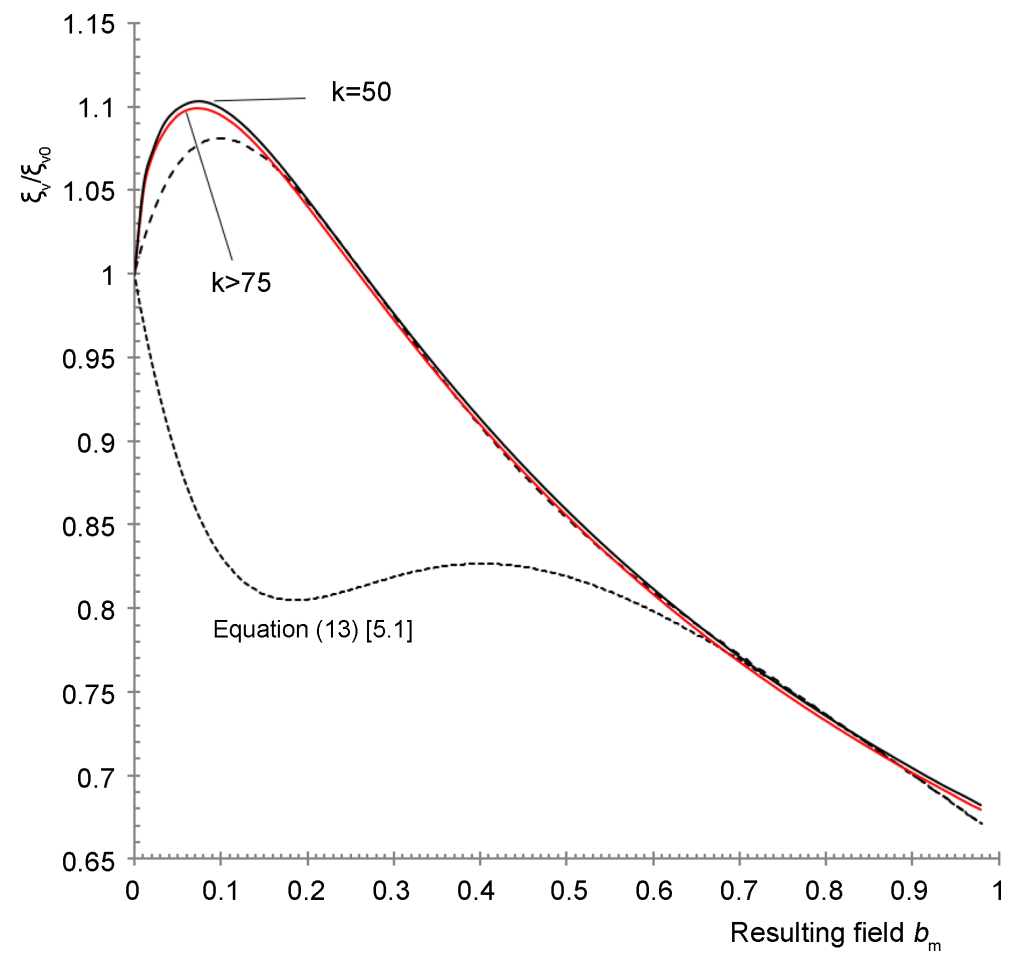

Figure 3. Calculated variational parameter $\xi_{v} / \xi_{v 0}$ vs. $\bar{b}$ at $k \geq 50$, the solid red line is cubic spline fit to the dots at $k=100$, see Annex; $\xi_{v 0}$ is from Equation (19), the dotted and the dashed lines with no markers are from [[8], Equation (13)] with the constants equal to “ -4.3 " and " +4.3 " respectively.

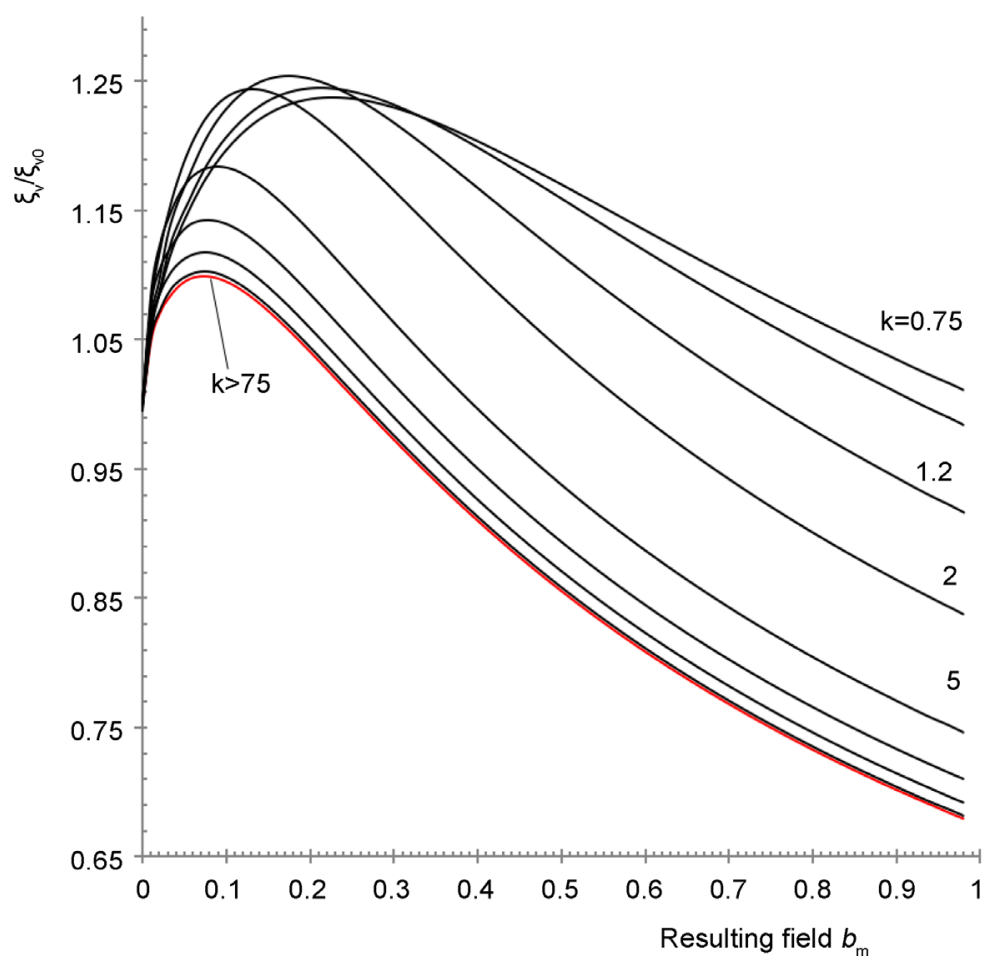

Figure 4. Calculated variational parameter $\xi_{v} / \xi_{v 0}$ vs. $\bar{b}$ at $k \leq 50$ : the solid black lines are cubic spline fits to the dots from Annex at $k=0.75,0.85,1.2,2,5,10,20$ and 50; $\xi_{v 0}$ is from Equation (19). The solid red line for $k \geq 75$ is given here as a reference. 
where $f_{r}^{\prime}=\mathrm{d} f_{r} / \mathrm{d} r ; F_{e m}$-is related to super-current and to magnetic field; the first term of $F_{\text {core }}$-to the local density of the Cooper pairs, and the second term: $\frac{\left(f_{r}^{\prime}\right)^{2}}{k^{2}}$-to their interaction [2] [8] [9] [10] [13]; $\pi R^{2}=2 \pi / k \bar{b}_{k}$ is the area of the circular (Wigner-Seitz) unit cell carrying one magnetic flux quantum, thus $R=\sqrt{2 / k \bar{b}_{k}} \quad f_{r}^{\prime}=d f_{r} / d r$ and Table A5 lists the scaling factors.

\subsection{Ginzburg-Landau Equations}

The GL equations are obtained by minimising the free energy of superconductor $F$ with respect to e.g., $f_{r}$ and to $q_{r}$ (form 1, Table 1 ) or to $f_{r}$ and $b_{k r}$ (form 2). In the circular cell approximation both the order parameter and magnetic flux density have axial symmetry and for this case two stationary GL equations in two forms are listed in Table 1, where the five local quantities: $f_{r}$ (with $\left.\Delta f_{r}=\frac{\mathrm{d}}{r \mathrm{~d} r}\left(\frac{r \mathrm{~d} f_{r}}{\mathrm{~d} r}\right)=\frac{\mathrm{d}^{2} f_{r}}{\mathrm{~d} r^{2}}+\frac{\mathrm{d} f_{r}}{r \mathrm{~d} r}=f_{r}^{\prime \prime}+f_{r}^{\prime} / r\right), \quad a_{r}=a_{\varphi}(r), \quad b_{k r}=b_{k z}(r)$, $q_{r}=q_{\varphi}(r)=a_{r}-1 / k r$ and $j_{r}=j_{\varphi}(r)$ are respectively moduli of the order parameter, of vector potential (satisfying the Coulomb gauge and having only $\varphi$-component [5]), of local magnetic flux density vector (having only $z$-component), of the dimensionless super-velocity (having only $\varphi$-component), and of the vortex current density vector (having only $\varphi$-component). The two unknowns are: $f_{r}$ and one of the following: $a_{r}$ or $b_{k r}$ respectively for the forms 1 or 2.

Complementing the Equations (1) (2) and (3) (4) Maxwell equations are:

$$
b_{k r}=\frac{\mathrm{d}\left(r q_{r}\right)}{r \mathrm{~d} r}=\frac{\mathrm{d}\left(r a_{r}\right)}{r \mathrm{~d} r} ; j_{r}=\frac{\mathrm{d} b_{k r}}{\mathrm{~d} r}=\frac{\mathrm{d}}{\mathrm{d} r}\left[\frac{\mathrm{d}\left(r q_{r}\right)}{r \mathrm{~d} r}\right]=q_{r}^{\prime \prime}+\frac{q_{r}^{\prime}}{r}-\frac{q_{r}}{r^{2}} \text {. }
$$

\section{Solving Ginsburg-Landau Equation(s)}

From Equations (4) and (5) one gets:

$$
\frac{r}{f_{r}^{2}}=\frac{\rho_{r}^{2}}{f_{\infty}^{2} r} \text { and } \frac{\mathrm{d}}{r \mathrm{~d} r}\left(\frac{\rho_{r}^{2}}{r} \frac{\mathrm{d} b_{k r}}{\mathrm{~d} r}\right)=f_{\infty}^{2} b_{k r}
$$

since $f_{\infty}^{2}$ is independent on $r$. Replacing the variables: $r \mathrm{~d} r=\rho_{r} \mathrm{~d} \rho_{r}$ in Equation (10) and assuming $\rho_{r} \neq 0, \quad f_{\infty} \neq 0$ gives:

$\frac{\mathrm{d}}{\rho_{r} \mathrm{~d} \rho_{r}}\left(\frac{\rho_{r} \mathrm{~d} b_{k r}}{\mathrm{~d} \rho_{r}}\right)=\frac{\mathrm{d}^{2} b_{k r}}{\mathrm{~d} \rho_{r}^{2}}+\frac{\mathrm{d} b_{k r}}{\rho_{r} \mathrm{~d} \rho_{r}}=f_{\infty}^{2} b_{k r}$, or: $\rho_{r}^{2} \frac{\mathrm{d}^{2} b_{k r}}{\mathrm{~d} \rho_{r}^{2}}+\rho_{r} \frac{\mathrm{d} b_{k r}}{\mathrm{~d} \rho_{r}}-\left(f_{\infty}^{2} \rho_{r}^{2}+0\right) b_{k r}=0$.

Since $\xi_{v} \neq 0$ (Section 3.4), also $\rho_{r} \neq 0$ everywhere, see Equation (3). On the other hand, Equation (11) is identical to Equation (4) everywhere except at $f_{\infty}=0$, which is the case at $\bar{b} \geq 1\left(\bar{b}_{k} \geq k\right)$. Therefore, I conclude that at $\bar{b} \rightarrow 1$ the approach [7] must complement [8] [9] [10] as further elaborated in Section 4.2. At $f_{\infty} \neq 0$ (and with $f_{\infty}$ independent on $r$ ) Equation (11) is the modified Bessel equation with the solution:

$$
b_{k r}=b_{k z}(r)=h_{k z}(r)=c_{1} I_{0}\left(f_{\infty} \rho_{r}\right)+c_{2} K_{0}\left(f_{\infty} \rho_{r}\right),
$$

where $h_{k z}(r)=H_{z}(r) / \sqrt{2} H_{c}$ is magnetic field strength, $I_{0}$ and $K_{0}$ are the modified Bessel's functions respectively of the first and second kind and order 0; 
$c_{1}$ and $c_{2}$ are the integration constants set by the boundary conditions. So far the solution is the same as obtained in [8] [9] [10]. Moreover, from Equation (12) one obtains:

$$
j_{r}=j_{\varphi}(r)=b_{k r}^{\prime}=\frac{f_{\infty} r \mathrm{~d} b_{k r}}{\rho_{r} \mathrm{~d}\left(f_{\infty} \rho_{r}\right)}=f_{\infty} r \rho_{r}^{-1}\left[c_{1} I_{1}\left(f_{\infty} \rho_{r}\right)-c_{2} K_{1}\left(f_{\infty} \rho_{r}\right)\right]
$$

where $I_{1}$ and $K_{1}$ are the modified Bessel's functions respectively of the first and second kind and order 1 . Note that the derivation of the solution (Equations (10)-(13)) was skipped in [8] [9] [10] and as a result, the important restriction $f_{\infty} \neq 0$ of the solution was hidden so far.

\subsection{Boundary Conditions}

The boundary conditions can be found e.g., in [3] [5] [6] [8]-[13]. From there a variety follows of the definitions for $c_{1}$ and $c_{2}$, such as based upon [8] [9] [10] [13]: 1) quantisation of magnetic flux through the unit cell: $\left.j_{r}\right|_{r \rightarrow 0}=-r f_{\infty}^{2} / k \xi_{v}^{2}$ and 2) zero current at its interface $j_{r}(R)=0$ :

$$
\begin{gathered}
c_{1}=\frac{f_{\infty}}{k \xi_{v}} \frac{K_{1}\left(f_{\infty} \rho_{R}\right)}{c_{0}}, c_{2}=\frac{f_{\infty}}{k \xi_{v}} \frac{I_{1}\left(f_{\infty} \rho_{R}\right)}{c_{0}}, \\
c_{0}=I_{1}\left(f_{\infty} \rho_{R}\right) K_{1}\left(f_{\infty} \xi_{v}\right)-I_{1}\left(f_{\infty} \xi_{v}\right) K_{1}\left(f_{\infty} \rho_{R}\right) .
\end{gathered}
$$

In order to stay focused and limit the size of the paper, below I simply accept Equations (14), (15) and will study namely this case in more detail. With the constants $c_{1}$ and $c_{2}$ defined, the solution allows calculating the local quantities as well as the mean quantities: equilibrium magnetic field, magnetisation, etc. In this paper I focus on magnetisation.

\subsection{Variational Method}

In the variational method Equation (5) in fact replaces the unknown $f(r)$ (which is otherwise obtained by solving the GL equations) and thus eliminates solving the first GL equation; instead one focuses on solving e.g., for $b_{k z}(r)$ (second GL equation) and for $f_{\infty}, \xi_{v}$. While drawbacks of the approach [5] [6] are treated in [8] [9] [10], I find that all three publications [8] [9] [10] are not easy to read as in turn they have crucial errors preventing the reader from wider use of this otherwise excellent method. For instance, in [8] [9] and [10] at least Equations (11), (13), (15); (22), (24), (28) and (2.18), (2.20) respectively have errors that can be decisive for the result. These errors are eliminated here and correct equations are presented thus facilitating broader use of the method. The analytical expressions for the energy density associated with the change in the order parameter near centers of vortices (Equations (15) [6] and (12) [8] respectively) though they look slightly different, are identical. On the other hand, Equation (20) of [9] is a substantial improvement of corresponding Equation (14) of [6] as it is explained in [8] [9] [10] (note that the corresponding Equation (11) [8] contains a typing error, the correct equations are: (2.16) in [10], (20) in [9] and Equation (16) here).

From Equation (12) at $r=0, h_{k r}(0)$ follows and since $F_{e m}=\bar{b}_{k} h_{k r}(0)$, one 
gets (from Equations (6)-(8), (14) (15) here) [5] [6] [8] [9] [10]:

$$
\begin{gathered}
F_{\text {em }}=\bar{b}_{k}\left[c_{1} I_{0}\left(f_{\infty} \xi_{v}\right)+c_{2} K_{0}\left(f_{\infty} \xi_{v}\right)\right], \\
F_{\text {core }}=\frac{\left(1-f_{\infty}^{2}\right)^{2}}{2}+\frac{\bar{b}_{1} f_{\infty}^{2}}{2}\left[\left(1-f_{\infty}^{2}\right) \ln \left(\frac{2}{\bar{b}_{1}}+1\right)+\frac{f_{\infty}^{2}}{2+\bar{b}_{1}}\right]+\frac{\bar{b}_{k} f_{\infty}^{2}\left(1+\bar{b}_{1}\right)}{k\left(2+\bar{b}_{1}\right)^{2}},
\end{gathered}
$$

with $\rho_{R}=\sqrt{R^{2}+\xi_{v}^{2}}$ and $\bar{b}_{1}=\bar{b}_{k} k \xi_{v}^{2}$.

Minimising the free energy density $F$ of superconductor, Equations (6)-(8), (16) (17), with respect to the variational parameters $f_{\infty}$ and $\xi_{v}$ (at fixed $k$ and $\bar{b}$ ) allows establishing dependencies of $f_{\infty}$ and $\xi_{v}$ on $k$ and $\bar{b}$ in a self-sufficient way [5] [6] [8] [9] [10], see detailed in Figures 1-4 and Annex. Once these are established, the order parameter is defined (Equation (5)) and the dependence of magnetisation on $k$ and $\bar{b}$ follows, see Equations (20)-(23) (24). In [5] [6] [8] [9] [10], the accurate dependencies of $f_{\infty}$ and $\xi_{v}$ on $k$ and $\bar{b}$ are missing, instead inaccurate interpolation fits are published in both cases as explained below and clear from Figures 1-4. This prevents accurate calculation of magnetisation in particular. In this paper the minimising of $F$ is done numerically (using Solver in Excel), results are presented in a clear, traceable form and in more detail as compared to [5] [6] [8] [9] [10] and they yield the following.

\subsection{Variational Parameter $f_{\infty}$}

At any $5 \leq k \leq 200$ all obtained data points of the dependence $f_{\infty}(\bar{b}, k)$ collapse at one curve fitted here by a cubic spline, see Figure 1 and Table A1. For this reason no data for the individual splines is given here for this range. This simplification causes an estimated error of $0.5 \%$ as explained in Section 5 and the error can be reduced by using more accurate data from the minimising of $F$. Furthermore, the obtained data are in reasonable agreement with that from [[8], Equation (14)] (numbered as Equation (18) here), except at lower

$(\bar{b}<0.25)$ and at higher $(\bar{b}>0.75)$ fields, see Figure 1 :

$$
f_{\infty}(\bar{b})=\left(1-\frac{\bar{b}^{2}}{2.8}\right)\left\{\left[1+1.7 \bar{b}(1-1.4 \bar{b})^{2}\right]\left[1-\left(\frac{\bar{b}}{0.985}\right)^{4}\right]\right\}^{1 / 2}
$$

At $k_{12} \leq k<5$ the obtained dots deviate from this curve and the error when using Equation (18) can reach $5 \%$ as Figure 1 exemplifies. Therefore in this range instead of using Figure 1 or Equation (18), I recommend using Figure 1 or more accurately the data from Annex e.g., by constructing the individual splines. Importance of this correction becomes clear in Section 5. For the above reasons I am convinced that Equation (18) does not approximate the dependence of $f_{\infty}(\bar{b}, k)$ with "an accuracy of about $0.5 \%$ for arbitrary $k$ and $b$ " as stated in [8] [9] [10]. In addition, I find unsatisfactory the agreement of the obtained data with the Equation (24) of [6]:

$f_{\infty}(\bar{b})=1-(\bar{b})^{4}$.

\subsection{Variational Parameter $\xi_{v}$}

Minimisation of $F$ with respect to $\xi_{v}$ at $\bar{b} \rightarrow 0$ shows that Equation (15) of 
[8] is less accurate than the original Equation (16) of [5], especially at $k<10$ (and that the error exceeds 1\% up to $k=50$ ), see Figure 2. Therefore, the correct equation to calculate the dependence $\xi_{v 0}(k)$ for $\bar{b} \rightarrow 0$ at any $k$ in an ideal type II superconductor is [5]:

$$
k \xi_{v 0}=\sqrt{2}\left[1-\frac{K_{0}^{2}\left(\xi_{v 0}\right)}{K_{1}^{2}\left(\xi_{v 0}\right)}\right]^{\frac{1}{2}} .
$$

The obtained (by minimizing $F$ ) dependence $\xi_{v}(\bar{b}, k)$ is similar and different from those described in [3] [5]. On one hand at $k \geq 75$ all data points collapse practically at one curve, see Figure 3 and Table A2 in Annex. For this reason only one cubic spline fit (namely, $k=100$ ) is shown, the error of this simplification is below $0.5 \%$.

The agreement of the obtained data with [[8], Equation (13)] is unsatisfactory, see Figure 3 and therefore I recommend calculating $\xi_{v}(\bar{b}, k)$ for $k \geq 75$ and any value of magnetic field $\bar{b}$ from the spline fit, Figure 3 and Table A2 in Annex.

On the other hand at $k<50$ the obtained dependence $\xi_{v}(\bar{b}, k)$ is rather different from those described in [5] [6] [8] [9] [10] and from the single curve $k \geq 75$ in Figure 3. For instance, the relative difference for $k=50$ and 0.75 reaches $50 \%$ as Figure 4 shows.

Based on this study, I conclude that none of the Equations (13)-(15) in [8] (repeated as (22)-(24) in [9] and as (2.18)-(2.20) in [10]) is accurate for arbitrary values of $k \geq k_{12}$ and over the entire range of magnetic fields $0 \leq \bar{b} \leq 1$. Instead I recommend using more accurate data from this paper, see Annex. The most accurate results are obtained through minimising the free energy density $F$ of superconductor with respect to the variational parameters $f_{\infty}$ and $\xi_{v}$ as described in this paper. This step is a must when aiming at the agreement better than $1 \%$ between magnetisation calculated symbolically and numerically, see Section 5 .

\section{Magnetisation (Derived Symbolically)}

\subsection{Variational Approach}

The applied (thermodynamic, equilibrium) magnetic field $b_{k a}\left(\bar{b}_{k}\right)=\frac{\partial F}{2 \partial \bar{b}_{k}}$ from Equations (14)-(17) according to [5] [6] [8] [9] [10] is:

$$
b_{k a}\left(\bar{b}_{k}\right)=b_{k a}^{e m}\left(\bar{b}_{k}\right)+b_{k a}^{\text {core }}\left(\bar{b}_{k}\right) \text {, }
$$

with [8]:

$$
\begin{gathered}
b_{k a}^{e m}\left(\bar{b}_{k}\right)=b_{k a}^{e m 1}\left(\bar{b}_{k}\right)+b_{k a}^{e m 2}\left(\bar{b}_{k}\right), \\
b_{k a}^{e m 1}\left(\bar{b}_{k}\right)=\frac{1}{2}\left[c_{1} I_{0}\left(f_{\infty} \xi_{v}\right)+c_{2} K_{0}\left(f_{\infty} \xi_{v}\right)\right], \\
b_{k a}^{e m 2}\left(\bar{b}_{k}\right)=\frac{c_{0}^{-2}}{2 k \bar{b}_{1} \rho_{R}^{2}},
\end{gathered}
$$




$$
b_{k a}^{\text {core }}\left(\bar{b}_{k}\right)=\frac{k f_{\infty}^{2} \xi_{v}^{2}}{2}\left[\frac{1-f_{\infty}^{2}}{2} \ln \left(1+\frac{2}{\bar{b}_{1}}\right)-\frac{1-f_{\infty}^{2}}{2+\bar{b}_{1}}+\frac{f_{\infty}^{2}}{\left(2+\bar{b}_{1}\right)^{2}}\right]+\frac{f_{\infty}^{2}\left(2+3 \bar{b}_{1}\right)}{2 k^{2}\left(2+\bar{b}_{1}\right)^{3}}
$$

and $\bar{b}_{1}=\bar{b}_{k} k \xi_{v}^{2}$. Since Equations (21)-(24) define the applied field $b_{k a}\left(\bar{b}_{k}\right)$, the magnetisation is:

$$
-m_{k 0}\left(\bar{b}_{k}\right)=b_{k a}\left(\bar{b}_{k}\right)-\bar{b}_{k} \text { or: }-m_{k 0}\left(b_{k a}\right)=b_{k a}-\bar{b}_{k}\left(b_{k a}\right), \quad m_{k 0}(\bar{b})=\frac{M_{0}(\bar{B})}{B_{c} \sqrt{2}}
$$

Finally, after re-normalisation of Equation (25), one obtains:

$$
-m_{0}(\bar{b})=b_{a}(\bar{b})-\bar{b} \text { with } m_{0}(\bar{b})=M_{0}(\bar{B}) / B_{c 2} .
$$

Namely the magnetization $-m_{0}(\bar{b})$ calculated from Equation (25) after correcting the error (see Section 4.2 ) becomes the magnetization $-m_{1}(\bar{b})$ compared in Section 5 to those calculated numerically and with other symbolic methods.

\subsection{The Error and the Correction}

The error (present in Equations (10)-(26) at $f_{\infty} \rightarrow 0$, see. Equations (10)-(11)) is evident from the Abrikosov solution of the linearized GL equations [7] stating:

$$
m_{4}=m_{4}\left(b_{a}, k\right)=\frac{b_{a}-1}{\left(2 k^{2}-1\right) \beta_{A}}
$$

for any $k$ and $\bar{b} \rightarrow 1$ with $\beta_{A}$ equal to 1.1803 (1.1596) for the vortex lattice with square (hexagonal) unit cells [4] and to 1.1576 for the lattice with circular cells [9]; $b_{a}=\frac{B_{a}}{B_{c 2}}, \quad m_{4}=\frac{M_{4}}{B_{c 2}}$. As the examples in Section 5 will illustrate, at $\bar{b} \rightarrow 1$ the line 5 ([8] [9] [10] representing the magnetisation calculated from Equations (25) (26) without corrections) crosses the line 4 representing the $m_{4}$ and moreover it crosses the horizontal axis at 0.985 (instead of at 1 as Equation (27) implies). The error in $m_{0}(\bar{b})$ is visible at least at $0.5 \leq b_{a} \leq 1$.

In this paper the error is eliminated in the following way. The Equations (21)-(26) are only valid at $f_{\infty} \neq 0$, see Equation (11). Otherwise, Equation (27) is the correct symbolic solution of GL equation(s) providing the missing additional conditions to Equations (21)-(26) and simply stating that in the plane $-m_{1}\left(b_{a}\right)$ the point $m_{c 2}$ on the true magnetisation curve $m_{1}$ has the following coordinates: $m_{c 2}(1,0)$, in other words: $\left.m_{1}\right|_{b_{a}=1}=0$ and at $\bar{b} \rightarrow 1$

$$
\frac{\mathrm{d} m_{4}}{\mathrm{~d} b_{a}}=\frac{m_{4}}{1-b_{a}}=\frac{-1}{\left(2 k^{2}-1\right) \beta_{A}},
$$

the $1^{\text {st }}$ derivative through this point of the true magnetisation curve should be constant set by Equation (28) and that only depends on $k$ and on the Abrikosov parameter $\beta_{A}>1$ [7]. Moreover, using Equation (30) and $F_{\min }$ (that follows from the minimising the free energy in Equations (16) (17)), we can now define more accurately the vague condition " $\bar{b} \rightarrow 1$ " (or " $1-b_{a} \ll 1$ " [3]) as:

$$
\bar{b}_{3} \leq \bar{b} \leq 1 \text { with } \bar{b}_{3} \text { being defined by the condition: } F_{\min }=F_{4} \text {. }
$$


From [[7], Equation (19)] it is clear that at $\bar{b}_{3} \leq \bar{b} \leq 1$ the free energy density (see Equations (6)-(8)) is (at minimum when $\beta_{A}$ is as close to 1 as possible):

$$
F_{4}=\left[\frac{1}{2}+\bar{b}_{k}^{2}-\frac{\left(1-\bar{b}_{k}\right)^{2}}{1+\left(2 k^{2}-1\right) \beta_{A}}\right] .
$$

From Equations (30) and (16) (17) it is easy to check that for $\beta_{A}=1.1576$, $k=200$ at $0.5 \leq \bar{b} \leq 1$ always $F_{\min }>F_{4}$, which means that in this range Equation (27) is more accurate than Equation (26). On the other hand, at $0 \leq \bar{b} \leq 0.5$ Equation (26) is more accurate than Equation (27), since here $F_{4}>F_{\min }$ (with $F_{\min }$ obtained by minimising $F$, see Equations (16) (17)). More generally, Equation (27) must be used at $\bar{b}_{3} \leq \bar{b} \leq 1$ instead of Equation (26).

A smooth transition from Equation (26) to Equation (27) can be achieved in several ways. In this paper I use the following approach. So far calculated from Equation (26) magnetisation $\left.m_{0}\right|_{b_{a}=0.985}=0$. Thus Equation (26) produces the erroneous value of the upper critical field $b_{c 2}^{*}$ (see Section 6.2) due to ignoring the restriction $f_{\infty} \neq 0$, see the condition by Equations (10) and (11). Replacing $\bar{b}$ by $\bar{b} / 0.985$ (Equation (33)) when plotting $m(\bar{b})$ satisfies the condition set by Equation (27) at $\bar{b} \rightarrow 1:\left.m_{0}\right|_{b_{a}=1}=0$. This step shifts the entire magnetisation curve (Equation (26)) parallel to itself and slightly to the right as exemplified in Section 5. Moreover, at $\bar{b} \rightarrow 1$ calculated from Equation (26) magnetisation should have the same first derivative as set by Equation (28). This is obviously not the case as one can see in Section 5 (not only magnetisation curve calculated from [8] [9] [10] crosses the horizontal axis at $b_{m}=0.985$, but it has the slope different from that set by Equation (27)).

In this paper a compliance with this condition (Equation (28)) is achieved by introducing the correction: $m_{1}=0.985 \cdot m_{0}(\bar{b})$, where $m_{0}(\bar{b})$ comes from Equation (26). This correction slightly rotates the entire magnetisation curve around the point $m_{c 2}(1,0)$, so that the derivative of the magnetisation $m_{1}$ becomes equal to that set by Equation (28) and the transition from $m_{1}$ to $m_{4}$ is smooth since the higher derivatives are preserved. Furthermore, used for the comparison (in Section 5) magnetisation $m_{2}$ from [[3], Figure 7] is calculated numerically for the triangular vortex lattice, while the obtained here results are for the vortex lattice of circular cells, therefore accounting the respective ratio of the Abrikosov parameters (Equation (27)), more accurately: $m_{1}=0.983 \cdot m_{0}(\bar{b})$ in this case. Clearly, more sophisticated methods of achieving the same result can be used (all required math for combining the solutions is present e.g., in [13]), but they are beyond the scope of this paper.

In conclusion, the correction makes the obtained solution compliant with [7] [13] (the same point $m_{c 2}$ and the same direction of the magnetisation curve at this point). It should be noted that this correction of the magnetisation uses the symbolic form of the theoretical result [7] at $\bar{b} \rightarrow 1$ and therefore the solution obtained here remains self-sufficient (even though it now uses two solutions of GL equations: [8] [9] [10] and [7]). So far I did not use any numerical results (the fact that calculated numerically magnetisation $m_{2}$ also agree with the conditions of Equations (27), (28) only means that these conditions are just). Cor- 
rected this way magnetisation $m_{1}$ is in excellent agreement with the conditions of Equations (27), (28) and is further compared to that calculated numerically (and with other symbolic methods) in Figures 5-8.

\section{Comparison and Discussion}

In Figures 5-8 the magnetisation $m_{1}$ is compared to that calculated numerically [3] and to those calculated symbolically [7] [8] [9] [10] through the entire range of magnetic fields $0 \leq \bar{b} \leq 1$ and representative range of the GL parameter $k_{12} \leq k \leq 200$ (same range as in [3]). In each figure the magnetisation changes between 0 and $-m_{c 1}=b_{c 1}$ (further defined in Section 6.1). Moreover, in this range of $k$ the $b_{c 1}$ changes by more than 4 orders in magnitude: between 1 and $7.2 \times 10^{-5}$. An overview can be found elsewhere [3] [4] of the general features of reversible magnetisation, of its dependence on $k$ and on magnetic field, etc. as follows from numerical solving of GL equations and these are not discussed here. Instead, I focus on the validation of the obtained analytical solution (Equations (20)-(26) complemented by Equations (27)-(30) and with the data in Figures 1-4 and Annex). This is achieved by comparing magnetization $m_{1}$ to magnetisation calculated using other methods. Namely, in Figures 5-8 I compare $m_{1}$ to $m_{2}-m_{5}$ being respectively magnetisation calculated from: this work $\left(m_{1}\right)$; [3] [4], numerically for hexagonal unit cells $\left(m_{2}\right)$; [[3], Equation (19)] from the interpolation fit for $m_{2}$ with limited validity $\left(m_{3}\right)$; [7], Equation (27) here $\left(m_{4}\right)$ and [8] [9] [10] as published $\left(m_{5}\right)$.

As clear from Figures 5-8, for any value of the GL parameter $k \geq k_{12}$ and over the entire range of magnetic fields $0 \leq b_{m}=\bar{b} \leq 1$ excellent agreement between $m_{1}$ and $m_{2}$ is achieved: the relative difference $\left|\frac{m_{1}-m_{2}}{m_{2}}\right|$ is below 1.5\% everywhere (except in the narrow range: $0 \leq b_{m} \leq 0.01$ where it is below $4 \%$, see further elaborated in Section 6.1).

\subsection{Ginzburg-Landau Parameter $k_{12} \leq k \leq 1$}

Representative for the range of $k_{12} \leq k \leq 1$ (with $0.58 \leq m_{c 1}=b_{c 1} \leq 1$, see [[3], Figure 1]) set of the magnetisation curves at $k=0.85$ is shown in Figure 5(a).

The relative difference $\left|\frac{m_{1}-m_{2}}{m_{2}}\right|$ is below $1.5 \%$ in the entire range

$0 \leq b_{m}=\bar{b} \leq 1$ (except in the range: $0 \leq b_{m} \leq 0.01$ where it is below $4 \%$, see further elaborated in Section 6.1). As expected [3], the interpolation fit $\left(m_{3}\right)$ totally fails to describe the data $\left(m_{2}\right)$ quantitatively, since $k<3$ and thus $\left|\frac{m_{3}-m_{2}}{m_{2}}\right|$ is too high. The data represented by magnetisation $m_{1}\left(\right.$ and $\left.m_{2}\right)$ are in good agreement with these represented by $m_{4}$ at $0.8 \leq \bar{b} \leq 1$ (so that both $\left|\frac{m_{1}-m_{4}}{m_{4}}\right|$ and $\left|\frac{m_{2}-m_{4}}{m_{4}}\right|$ e.g., at $\bar{b}=0.8$ are below $0.7 \%$, even though $\bar{b}_{3}=1$ as $k<1$ in 


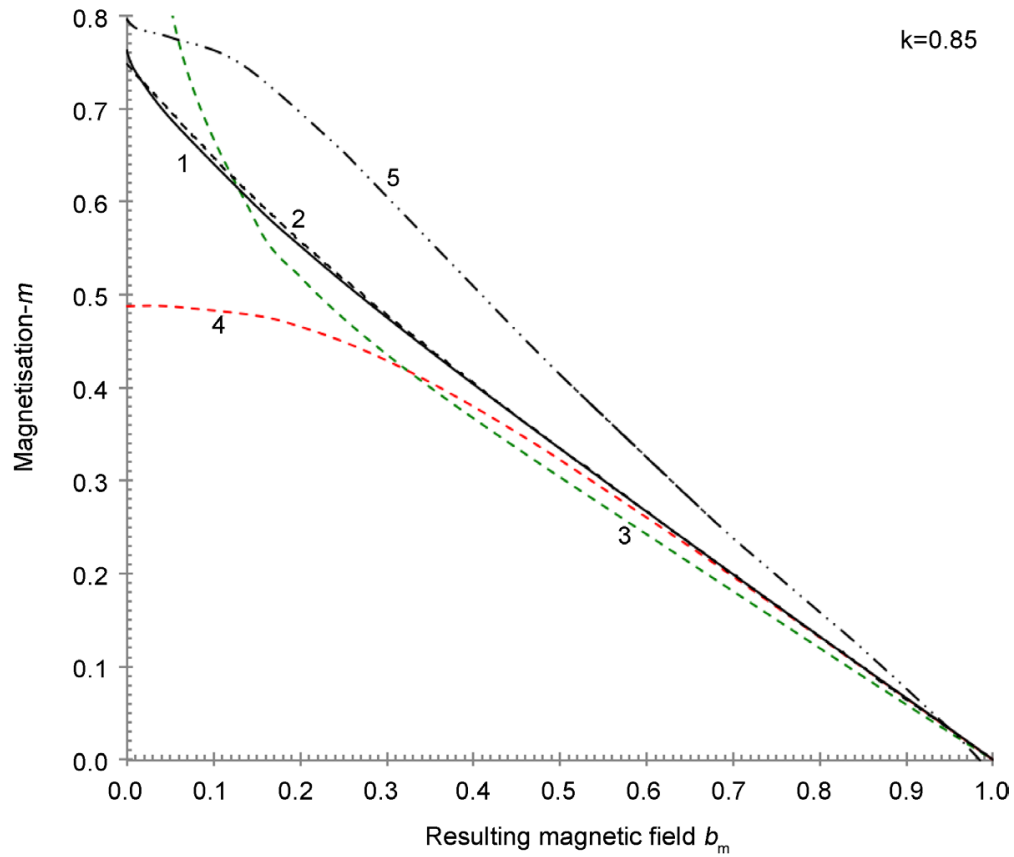

(a)

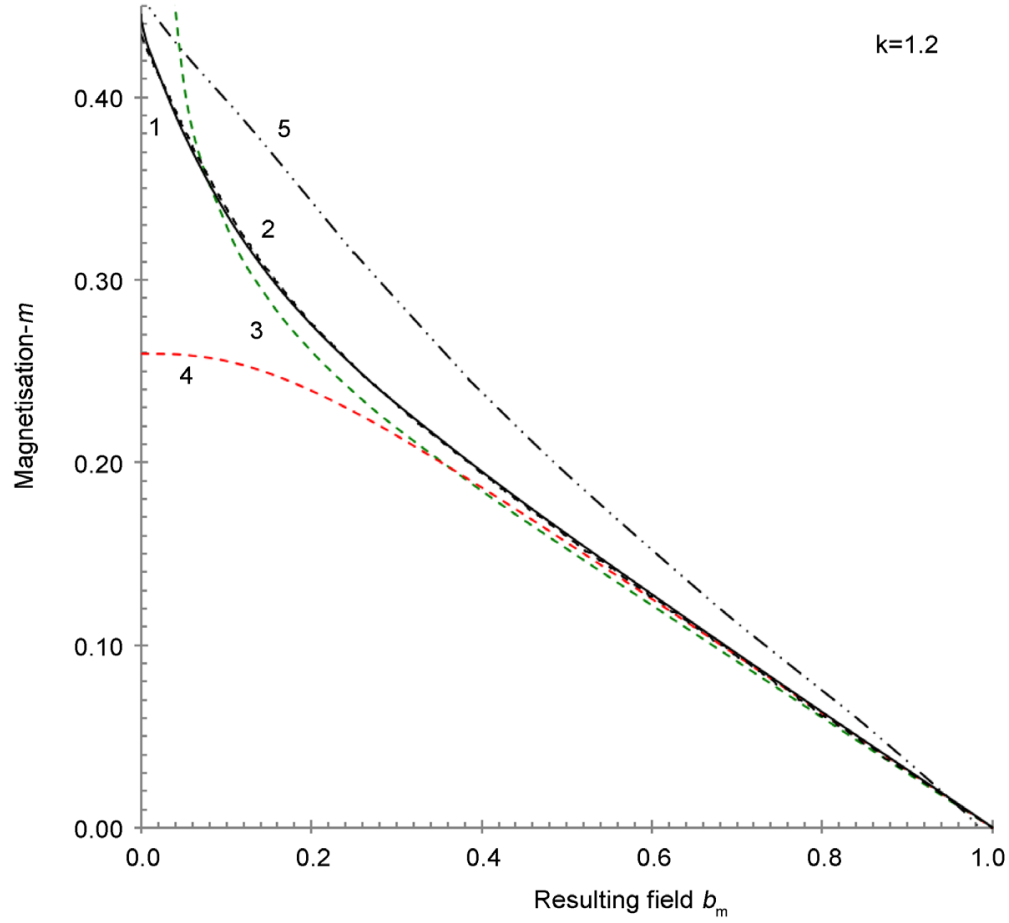

(b)

Figure 5. (a). Calculated for $k=0.85$ magnetisation $m$ as function of the resulting field $\bar{b}=b_{m}: 1$-as proposed here $\left(m_{1}\right) ; 2,3$-from the numerical solution, the dashed black line $\left(m_{2}\right)$ restored from [[3], Figure 7] and the dashed green line $\left(m_{3}\right)$ from the fit- [[3], Equation (19)]; 4-from Equation (27) $\left(m_{4}\right)$; 5-from [8] [9] [10] (dashed double dotted line, $\left.m_{5}\right)$. (b). Calculated for $k=1.2$ magnetisation $m$ as function of the resulting field $\bar{b}=b_{m}: 1$-as proposed here $\left(m_{1}\right) ; 2,3$-from the numerical solution, the dashed black line $\left(m_{2}\right)$ restored from [[3], Figure 7] and the dashed green line $\left(m_{3}\right)$ from the fit- [[3], Equation (27)]; 4-from Equation (27) $\left(m_{4}\right) ; 5$-from [8] [9] [10] (dashed double dotted line, $\left.m_{5}\right)$. The line numbering is the same as in Figure $5(\mathrm{a})$. 


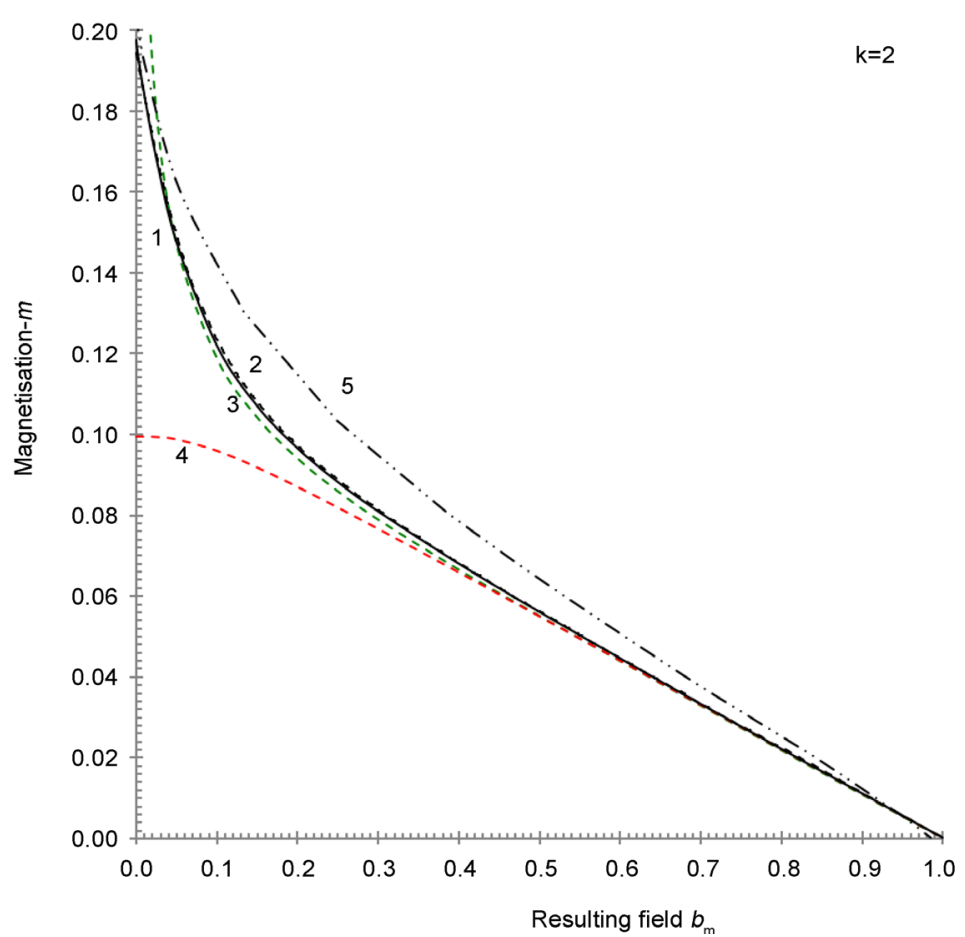

(a)

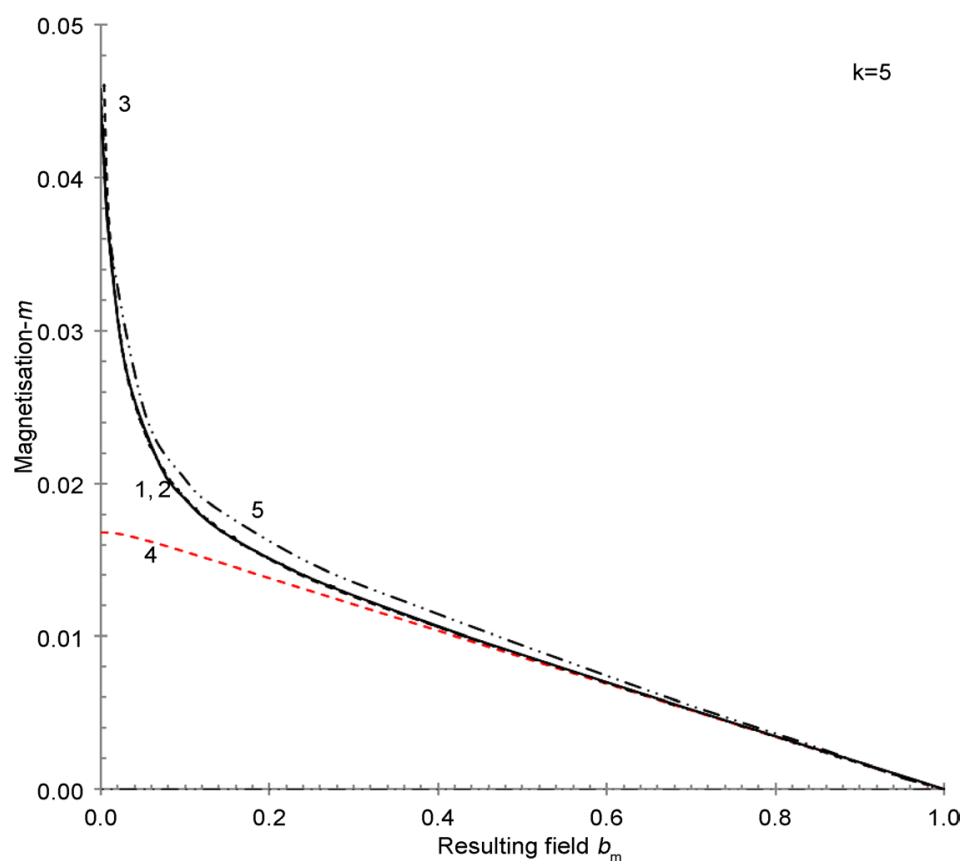

(b)

Figure 6. (a) Calculated for $k=2$ magnetisation $m$ as function of the field $\bar{b}=b_{m}: 1$-as proposed here $\left(m_{1}\right) ; 2,3$-from the numerical solution, the dashed black line $\left(m_{2}\right)$ restored from [[3], Figure 7] and the dashed green line $\left(m_{3}\right)$ from the fit-[[3], Equation (19)]; 4-from Equation (27) $\left(m_{4}\right)$; 5-from [8] [9] [10] (dashed double dotted line, $\left.m_{5}\right)$. The line numbering is the same as in Figure 5(a). (b). Calculated for $k=5$ magnetisation $m$ as function of the field $\bar{b}=b_{m}: 1$-as proposed here $\left(m_{1}\right) ; 2,3$-from the numerical solution, the dashed black line $\left(m_{2}\right)$ restored from [[3], Figure 7] and the dashed green line $\left(m_{3}\right)$ from the fit-[[3], Equation (19)]; 4-from Equation (27) $\left(m_{4}\right) ; 5$-from [8] [9] [10] (dashed double dotted line, $m_{5}$ ). The line numbering is the same as in Figure 5(a). 


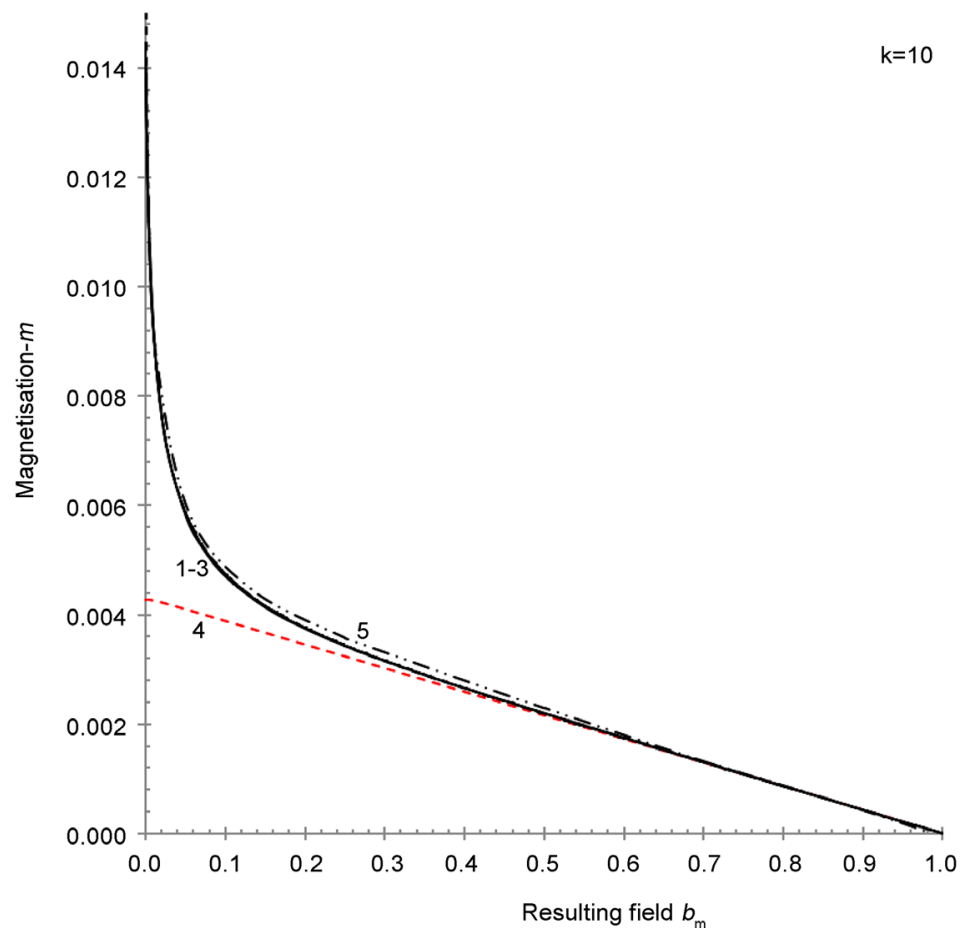

(a)

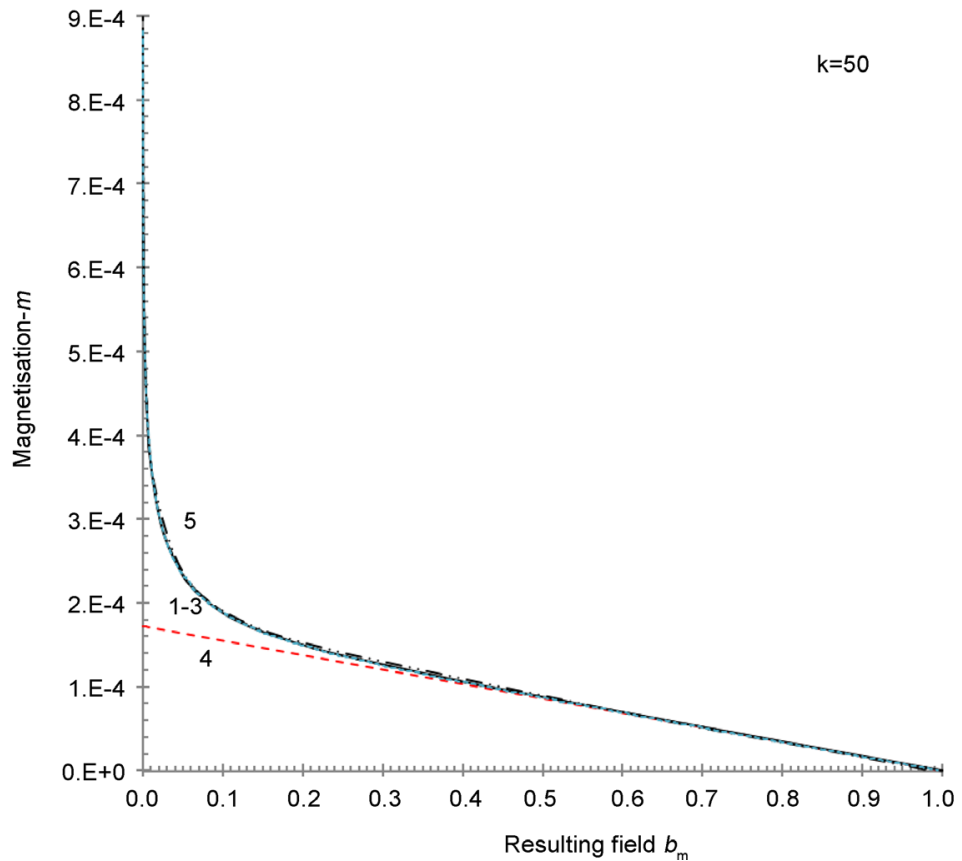

(b)

Figure 7. (a) Calculated for $k=10$ magnetisation $m$ as function of the field $\bar{b}=b_{m}: 1$-as proposed here $\left(m_{1}\right) ; 2,3$-from the numerical solution, the dashed black line $\left(m_{2}\right)$ restored from [[3], Figure 7] and the dashed green line $\left(m_{3}\right)$ from the fit-[[3], Equation (19)]; 4-from Equation (27) $\left(m_{4}\right)$; 5-from [8] [9] [10] (dashed double dotted line, $\left.m_{5}\right)$. The line numbering is the same as in Figure 5(a). (b) Calculated for $k=50$ magnetisation $m$ as function of the field $\bar{b}=b_{m}: 1$-as proposed here $\left(m_{1}\right) ; 2,3$-from the numerical solution, the dashed black line $\left(m_{2}\right)$ restored from [[3], Figure 7] and the dashed green line $\left(m_{3}\right)$ from the fit-[[3], Equation (19)]; 4-from Equation (27) $\left(m_{4}\right)$; 5-from [8] [9] [10] (dashed double dotted line, $m_{5}$ ). The line numbering is the same as in Figure 5(a). 


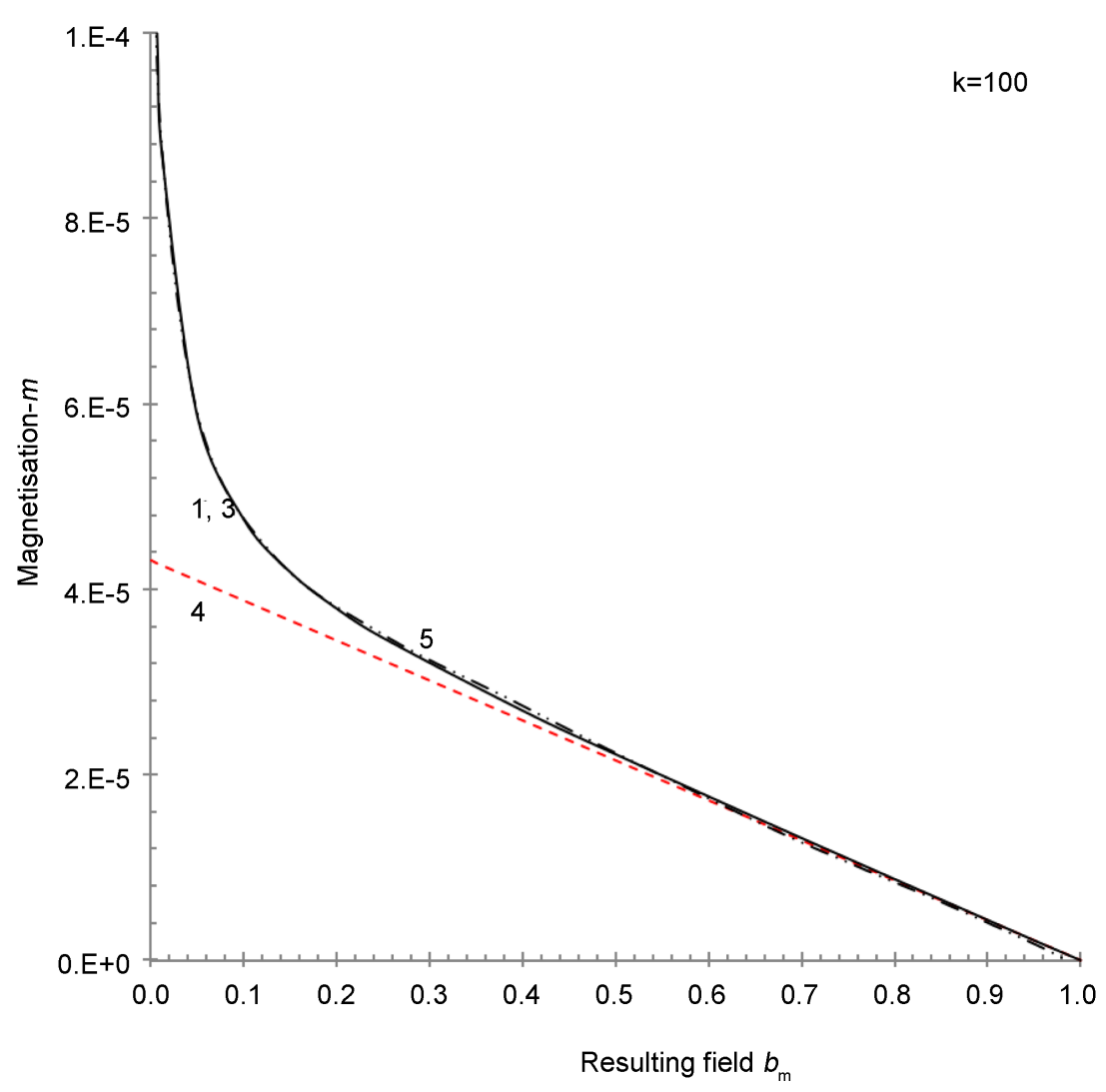

(a)

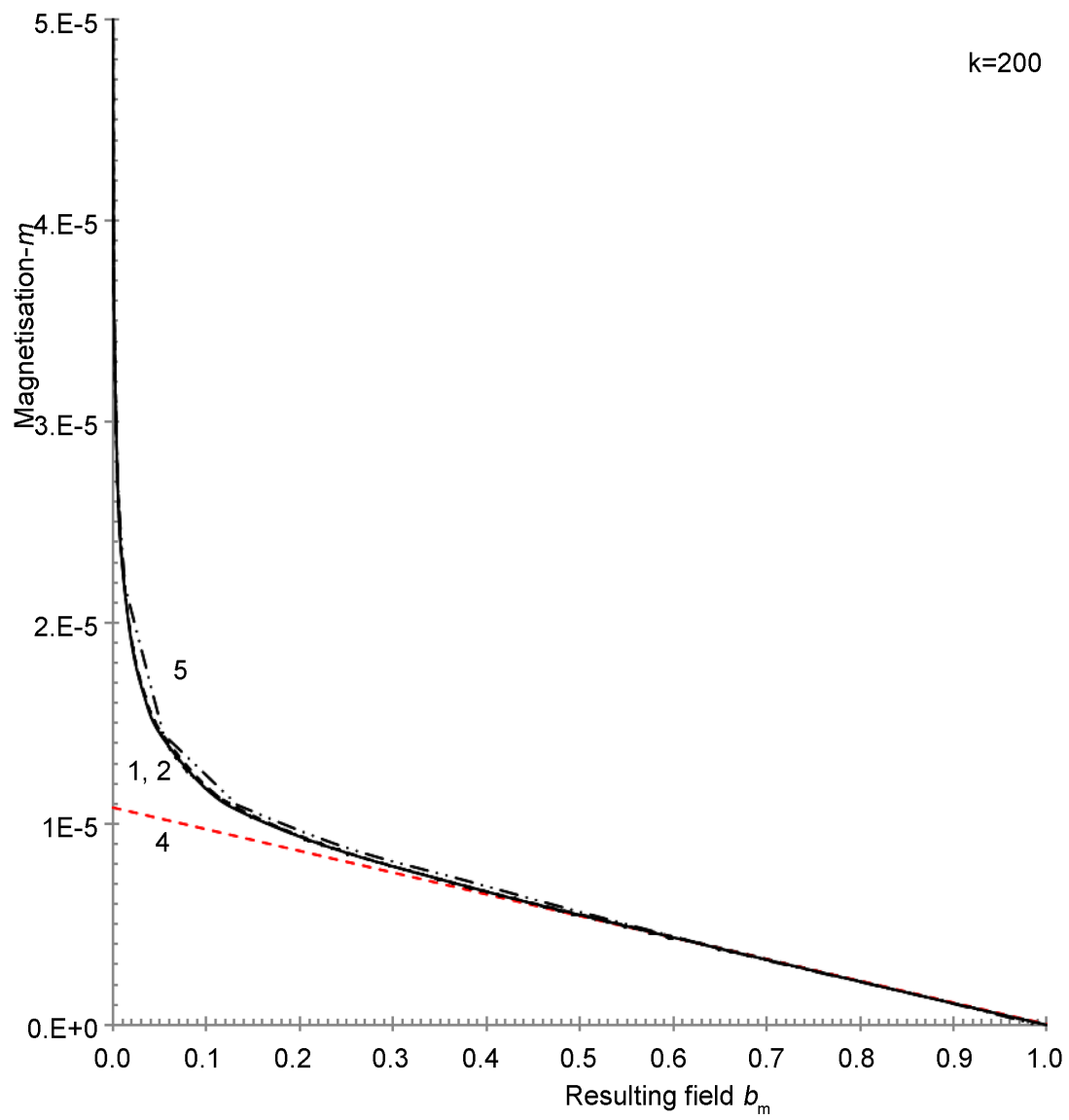

(b) 


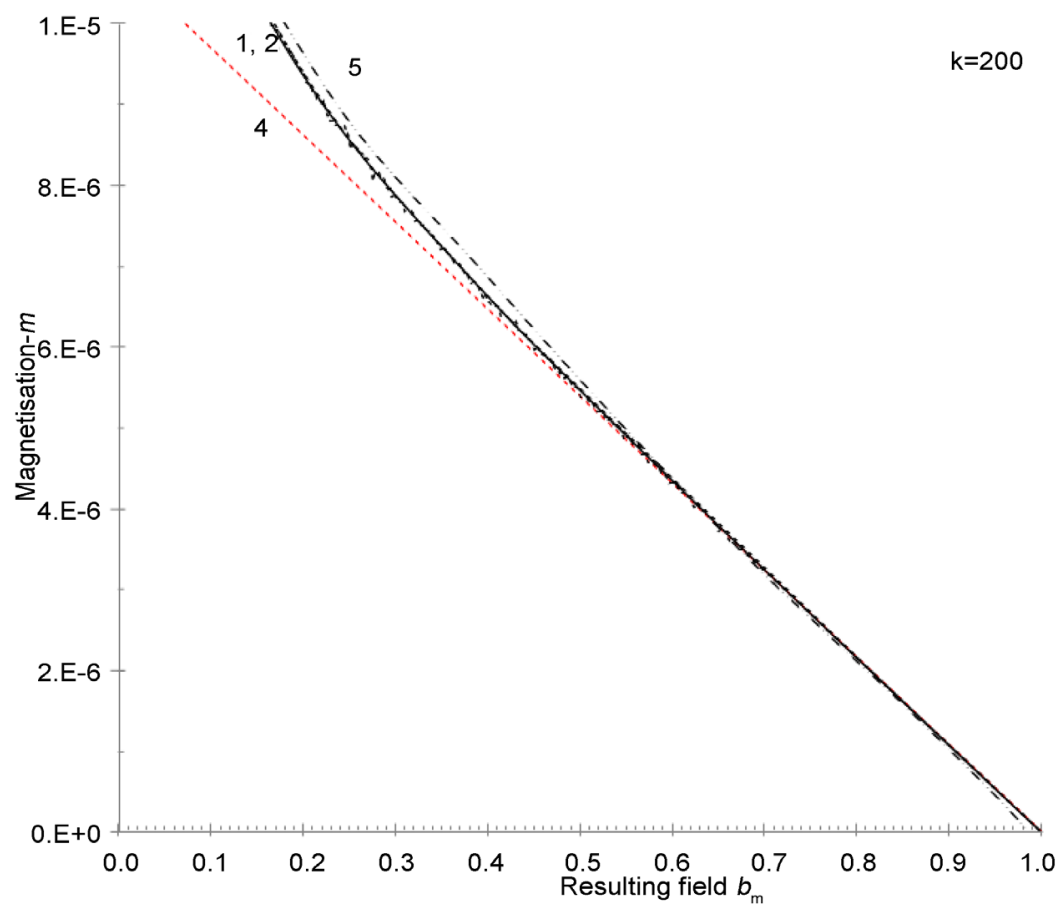

(c)

Figure 8. (a) Calculated for $k=100$ magnetisation $m$ as function of the field $\bar{b}=b_{m}$ : 1 -as proposed here $\left(m_{1}\right) ; 2,3$-from the numerical solution, the dashed black line $\left(m_{2}\right)$ restored from [[3], Figure 7] and the dashed green line $\left(m_{3}\right)$ from the fit-[[3], Equation (19)]; 4-from Equation (27) $\left(m_{4}\right)$; 5-from [8] [9] [10] (dashed double dotted line, $m_{5}$ ). The line numbering is the same as in Figure 5(a). (b) Calculated for $k=200$ magnetisation $m$ as function of the field $\bar{b}=b_{m}: 1$-as proposed here $\left(m_{1}\right) ; 2,3$-from the numerical solution, the dashed black line $\left(m_{2}\right)$ restored from [[3], Figure 7] and the dashed green line $\left(m_{3}\right)$ from the fit-[[3], Equation (19)]; 4-from Equation (27) $\left(m_{4}\right)$; 5-from [8] [9] [10] (dashed double dotted line, $m_{5}$ ). The line numbering is the same as in Figure 5(a). (c) Fragment of the calculated for $k=200$ magnetisation $\mathrm{m}$ as function of the field $\bar{b}=b_{m}$, see Figure 8(b) for more. The line numbering is the same as in Figure 5(a).

this case). It is clear from the figure that $m_{5}$ also fails to describe quantitatively the data represented by $m_{1}$ (and $m_{2}$ ): e.g., at $b_{m}=0.25$ the relative difference $\left|\frac{m_{5}-m_{2}}{m_{2}}\right|$ is $26.5 \%$, which is not competitive and not what one expects after reading [8] [9] [10].

\subsection{Ginzburg-Landau Parameter $1 \leq k \leq 2$}

Representative for the range of $1 \leq k \leq 2$ (with $0.2 \leq m_{c 1}=b_{c 1} \leq 0.58$, see [[3], Figure 1]) set of the magnetisation curves $k=1.2$ and $k=2$ is shown in Figure 5(b) and Figure 6(a) respectively.

In Figure 5(b) and Figure 6(a) the relative differences $\left|\frac{m_{1}-m_{2}}{m_{2}}\right|$ are below $1.1 \%$ and $1.3 \%$ respectively in the entire range $0 \leq b_{m}=\bar{b} \leq 1$ (except in the range: $0 \leq b_{m} \leq 0.01$ where they are below $4 \%$ and $3.2 \%$ respectively, as further elaborated in Section 6.1). As expected [3], the interpolation fit $\left(m_{3}\right)$ fails to de- 
scribe the data $\left(m_{2}\right)$ quantitatively, since $k<3$ and thus $\left|\frac{m_{3}-m_{2}}{m_{2}}\right|$ is high. The data represented by $m_{1}$ (and $m_{2}$ ) are in good agreement with these represented by $m_{4}$ at $0.8 \leq \bar{b} \leq 1$ (so that both $\left|\frac{m_{1}-m_{4}}{m_{4}}\right|$ and $\left|\frac{m_{2}-m_{4}}{m_{4}}\right|$ e.g., at $\bar{b}=0.8$ are below $0.4 \%$ (Figure $5(\mathrm{~b})$ ) and below $0.2 \%$ (Figure $6(\mathrm{a})$ ), with $\bar{b}_{3}=0.94$ and 0.75 respectively). It is clear from the figures that $m_{5}$ fails to describe quantitatively the data represented by $m_{1}$ (and $m_{2}$ ): e.g., at $b=0.25$ the relative difference $\left|\frac{m_{5}-m_{2}}{m_{2}}\right|$ is $24.5 \%$ (Figure 5(b)) and 16.1\% (Figure 6(a)), which is not competitive and not what one expects after reading [8] [9] [10].

\subsection{Ginzburg-Landau Parameter $2 \leq k \leq 5$}

Representative for the range of $2 \leq k \leq 5$ (with $0.045 \leq m_{c 1}=b_{c 1} \leq 0.2$, see [[3], Figure 1]) set of the magnetisation curves $k=2$ and $k=5$ is shown in Figure 6(a) and Figure 6(b) respectively. In Figure 6(b) the relative difference $\left|\frac{m_{1}-m_{2}}{m_{2}}\right|$ is below $0.5 \%$ in the entire range of magnetic fields $0 \leq b_{m}=\bar{b} \leq 1$ (except in the range: $0 \leq b_{m} \leq 0.01$ where it is below $2 \%$ respectively, as further elaborated in section 6.1). As expected [3] the interpolation fit $\left(m_{3}\right)$ describes the data $\left(m_{2}\right)$ quantitatively, since $k>3$ and thus $\left|\frac{m_{3}-m_{2}}{m_{2}}\right|$ is small, so both are represented by almost the same line in the figure. The data represented by $m_{1}$ (and $m_{2}$ ) are in good agreement with these represented by $m_{4}$ at $0.7 \leq \bar{b} \leq 1$ (so that both $\left|\frac{m_{1}-m_{4}}{m_{4}}\right|$ and $\left|\frac{m_{2}-m_{4}}{m_{4}}\right|$ e.g., at $\bar{b}=0.7$ are below $0.3 \%$, with $\bar{b}_{3}=0.6$ ). It is clear from Figure $6(\mathrm{~b})$ that $m_{5}$ still fails to describe quantitatively the data represented by $m_{1}$ (and $m_{2}$ ): e.g., at $b_{m}=0.25$ the relative difference $\left|\frac{m_{5}-m_{2}}{m_{2}}\right|$ is $7 \%$, which is not competitive and not what one expects after reading [8] [9] [10].

\subsection{Ginzburg-Landau Parameter $5 \leq k \leq 10$}

Representative for the range of $5 \leq k \leq 10$ (with $0.014 \leq m_{c 1}=b_{c 1} \leq 0.045$, see [[3], Figure 1]) set of the magnetisation curves at $k=5$ and $k=10$ is shown in Figure $6(\mathrm{~b})$ and Figure $7(\mathrm{a})$ respectively. In Figure $7(\mathrm{a})$ the relative difference $\left|\frac{m_{1}-m_{2}}{m_{2}}\right|$ is below $1 \%$ in the entire range $0 \leq b_{m}=\bar{b} \leq 1$ (except in the range: $0 \leq b_{m} \leq 0.01$ where it is below $1.3 \%$ respectively, as further elaborated in section 6.1). As expected the interpolation fit $\left(m_{3}\right)$ describes the data $\left(m_{2}\right)$ quantitatively, since $k>3$ and thus $\left|\frac{m_{3}-m_{2}}{m_{2}}\right|$ is small [3] so both are represented by 
almost the same line in the figure. The data represented by $m_{1}$ (and $m_{2}$ ) are in good agreement with these represented by $m_{4}$ at $0.7 \leq \bar{b} \leq 1$ (so that both $\left|\frac{m_{1}-m_{4}}{m_{4}}\right|$ and $\left|\frac{m_{2}-m_{4}}{m_{4}}\right|$ e.g., at $\bar{b}=0.7$ are below $0.4 \%$, with $\left.\bar{b}_{3}=0.54\right)$. It is clear from Figure 7 (a) that $m_{5}$ describes quantitatively the data represented by $m_{1}$ (and $m_{2}$ ): with larger relative difference $\left|\frac{m_{5}-m_{2}}{m_{2}}\right|$ of e.g., $4 \%$ at $b=0.25$ (as compared to $\left|\frac{m_{1}-m_{2}}{m_{2}}\right|$ ) and with visible error in the magnetisation's first derivative (on $\bar{b}$ ).

\subsection{Ginzburg-Landau Parameter $10 \leq k \leq 50$}

Representative for the range of $10 \leq k \leq 50$ (with $8.8 \times 10^{-4} \leq m_{c 1}=b_{c 1} \leq 0.014$ ) set of the magnetisation curves at $k=10$ and $k=50$ is shown in Figure 7(a) and Figure 7(b) respectively. In Figure 7(b) the relative difference $\left|\frac{m_{1}-m_{2}}{m_{2}}\right|$ is below $0.6 \%$ in the entire range $0 \leq b_{m}=\bar{b} \leq 1$. As expected the interpolation fit $\left(m_{3}\right)$ describes the data $\left(m_{2}\right)$ quantitatively, since $k>3$ and thus $\left|\frac{m_{3}-m_{2}}{m_{2}}\right|$ is small [3] so both are represented by almost the same line in the figure. The data represented by $m_{1}$ (and $m_{2}$ ) are in good agreement with these represented by $m_{4}$ at $0.7 \leq \bar{b} \leq 1$ (so that both $\left|\frac{m_{1}-m_{4}}{m_{4}}\right|$ and $\left|\frac{m_{2}-m_{4}}{m_{4}}\right|$ e.g., at $\bar{b}=0.7$ are below $0.5 \%$, with $\overline{b_{3}}=0.51$ ). It is clear from Figure 7 (a) that $m_{5}$ describes quantitatively the data represented by $m_{1}$ (and $m_{2}$ ): with larger relative difference $\left|\frac{m_{5}-m_{2}}{m_{2}}\right|$ of e.g., $3 \%$ at $b=0.25$ (as compared to $\left|\frac{m_{1}-m_{2}}{m_{2}}\right|$ ) and the error in the magnetisation first derivative (on $\bar{b}$ ) is present.

\subsection{Ginzburg-Landau Parameter $50 \leq k \leq 200$}

Representative for the range of $50 \leq k \leq 200$ (with

$7.2 \times 10^{-5} \leq m_{c 1}=b_{c 1} \leq 8.8 \times 10^{-4}$ ) set of the magnetisation curves at $k=100$ and $k=200$ is shown in Figure 8(a) and Figure 8(b) respectively. Since the accurate numerical data for $k=100$ are absent [3], we use $m_{3}$ instead of $m_{2}$ in Figure 8(a). The relative difference $\left|\frac{m_{1}-m_{3}}{m_{3}}\right|$ is below $1 \%$ (Figure 8(a)) and $\left|\frac{m_{1}-m_{2}}{m_{2}}\right|$ below $0.6 \%$ (Figure $\left.8(\mathrm{~b})\right)$ in the entire range $0 \leq b_{m}=\bar{b} \leq 1$. The data in Figure 8(a) represented by $m_{1}$ (and $m_{3}$ ) are in good agreement with these represented by $m_{4}$ at $0.7 \leq \bar{b} \leq 1$ (so that both $\left|\frac{m_{1}-m_{4}}{m_{4}}\right|$ and $\left|\frac{m_{3}-m_{4}}{m_{4}}\right|$ e.g., at $\bar{b}=0.75$ are below $1 \%$, with $\bar{b}_{3}=0.52$ ). The data in Figure $8(\mathrm{~b})$ represented 
by $m_{1}\left(\right.$ and $m_{2}$ ) are in good agreement with these represented by $m_{4}$ at $0.7 \leq \bar{b} \leq 1$ (so that both $\left|\frac{m_{1}-m_{4}}{m_{4}}\right|$ and $\left|\frac{m_{2}-m_{4}}{m_{4}}\right|$ e.g., at $\bar{b}=0.7$ are below $0.3 \%$, with $\left.\bar{b}_{3}=0.5\right)$. It is clear from Figure $8(a)$ that $m_{5}$ describes quantitatively the data represented by $m_{1}$ (and $m_{3}$ ) with larger relative difference $\left|\frac{m_{5}-m_{2}}{m_{2}}\right|$ of e.g., $2 \%$ at $b=0.25$ (as compared to $\left|\frac{m_{1}-m_{3}}{m_{3}}\right|$ ) and the error in the magnetisation $1^{\text {st }}$ derivative (on $\bar{b}$ ) is present. It is clear from Figure $8(\mathrm{~b})$ that $m_{5}$ describes quantitatively the data represented by $m_{1}$ (and $m_{2}$ ) with larger relative difference $\left|\frac{m_{5}-m_{2}}{m_{2}}\right|$ of e.g., $4 \%$ at $b=0.25$ (as compared to $\left|\frac{m_{1}-m_{2}}{m_{2}}\right|$ ) and the error in the magnetisation's first derivative (on $\bar{b}$ ) is present.

\subsection{Ginzburg-Landau Parameter $k=200$}

The fragment of the magnetisation curve at $k=200$ exemplifies that the error in the $1^{\text {st }}$ derivative of the magnetization $m_{5}$ is present at highest values of $k$ and results in the noticeable difference $\left|\frac{m_{5}-m_{1}}{m_{1}}\right|$. The noise of the magnetisation $m_{2}$ caused by the digitalisation of the data [[3], Figure 7] is also visible in the figure.

To summarise, over the entire ranges of the GL parameter $k_{12} \leq k \leq 1000$ and of magnetic field $0 \leq b_{m}=\bar{b} \leq 1$ excellent agreement between $m_{1}$ and $m_{2}$ is achieved: the relative difference $\left|\frac{m_{1}-m_{2}}{m_{2}}\right|$ is below $1.5 \%$ everywhere (except at the narrow range: $0 \leq b_{m} \leq 0.01$ where it is below $4 \%$ as elaborated in section 6.1). This result validates the advanced symbolic approach of this paper. On the other hand, for the first time numerical results [3] for hexagonal and circular unit cells are accurately validated over the entire ranges of $k$ and $b_{m}$ in a transparent way with the (essentially independent) symbolic method of solving GL equations. Moreover, I find that the obtained close agreement of $m_{1}$ and $m_{2}$ makes some of the interpolation fits [[3], Equations (15)-(23)] obsolete. Clearly, the remaining discrepancy of $m_{1}$ and $m_{2}$ can be reduced by expanding the data sets in Annex (the simplification error is now about 0.5\%), making the direct comparison of the underlying data, revising the symbolic solution and the boundary conditions, etc. This however is beyond the scope of this paper. It must be noted, that magnetisation calculated from Equations (20)-(26) is rather sensitive to the errors in calculating the variational parameters. Therefore, in order to avoid the interpolation errors in calculating $f_{\infty}$ and $\xi_{v}$ (that can be caused e.g., by splines), values of $f_{\infty}, \xi_{v}$ and $m$ are best calculated at exactly the same values of $\bar{b}$ and $k$.

Presented in Annex data for the dependencies of $f_{\infty}$ and $\xi_{v}$ on $k$ and $\bar{b}$ contain the simplification error of up to $0.5 \%$ (caused by the limit in the size of the paper). I recommend that when aiming at the agreement better than $99 \%$ 
between magnetisation calculated symbolically and numerically, one has to use more accurate data obtained through the minimising the free energy density $F$ of superconductor with respect to the variational parameters $f_{\infty}$ and $\xi_{v}$. Thus obviously "In order to achieve self-consistency in the theory the dependencies $\xi_{v}(k, \bar{b})$ and $f_{\infty}(k, \bar{b})$ should be obtained by numerically minimising the function $F\left(k, \bar{b}, \xi_{v}, f_{\infty}\right)$ with respect to $\xi_{v}$ and $f_{\infty}$ " [8] [9] [10], to which I add: the minimisation procedure is straightforward, use Equations (6) (7) (8), (16) (17) and a standard optimisation program, such as commonly available Solver in Excel. I find the quote above missing the "how" part (almost as to write: in order to get solution of GL equations, solve them).

\section{The Critical Fields}

\subsection{The Lower Critical Field $b_{c 1}$}

In this paper I calculate values of the field $b_{c 1}$ from Equations (25) (26) typically at $\bar{b}<1 \times 10^{-6}$, since at $\bar{b}=0$ the Equation (25) diverges. In Figure 9 the boxes show calculated this way dependence $b_{c 11}(k)$ of the lower critical field $b_{c 1}$. The black solid line is calculated from the fit to the numerical results [3] [4]:

$$
b_{c 12}=\left\{\ln (k)+\alpha_{\infty}+\exp \left[-c_{0}-c_{1} \ln (k)-c_{2} \ln ^{2}(k)\right]\right\}
$$

with $\quad \alpha_{\infty}=0.49693 ; \quad c_{0}=0.41477 ; \quad c_{1}=0.775 ; \quad c_{2}=0.1303$.

The red dashed line is calculated from the symbolic expression for isolated flux line [5] [6]:

$$
b_{c 13}=\left(\xi_{v 0}^{2}+k^{-2}\right) / 8+K_{0}\left(\xi_{v 0}\right) /\left[2 k^{2} \xi_{v 0} K_{1}\left(\xi_{v 0}\right)\right] .
$$

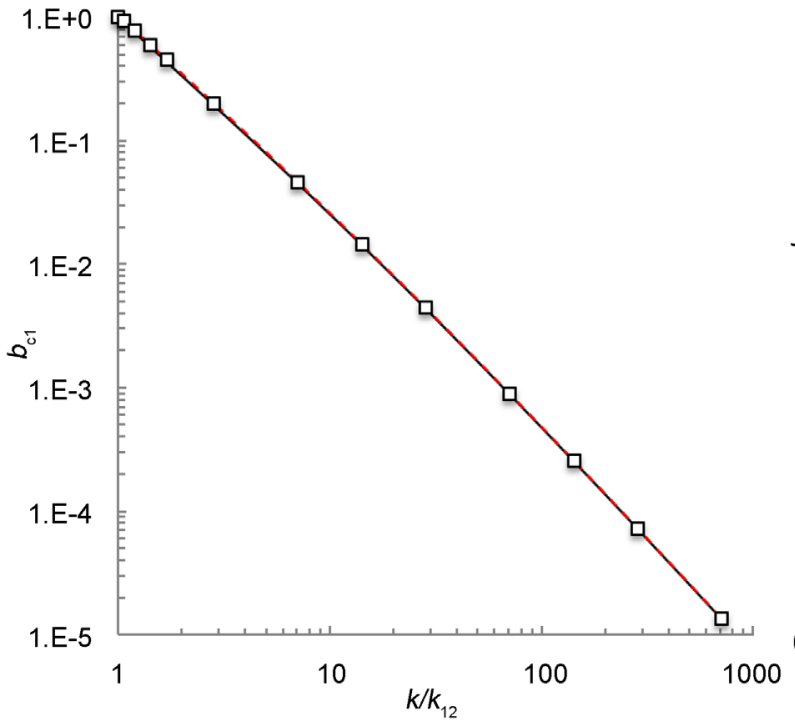

(a)

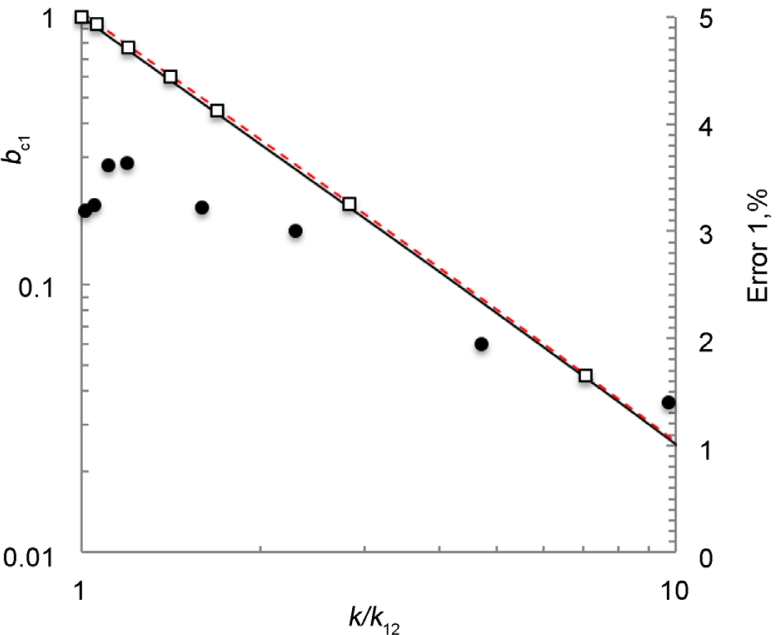

(b)

Figure 9. Calculated dependence on $k$ of the lower critical field $b_{c 1}$, (a): boxes-this paper $\left(b_{c 10}\right)$; the solid black line-numerically, Equation (31) $\left(b_{c 12}\right)$; the dashed line-Equation (32) $\left(b_{c 13}\right)$; (b): fragment of the same, the black circles-the relative "error", \%: $\frac{b_{c 11}-b_{c 12}}{b_{c 12}}$. 
In this range of $k$ the $b_{c 1}$ changes by 5 orders of magnitude, so I find the agreement between $b_{c 11}, b_{c 12}$ and $b_{c 13}$ reasonable (at $k<0$ there is still room for improvement).

\subsection{The Upper Critical Field $b_{c 2}$}

As noted [8] [9] [10], and clear from Figures 5-8 and Annex, the value of the upper critical field $b_{c 2}^{*}$ following from the symbolic approach [8] [9] [10] is $1.5 \%$ lower than the true value of $b_{c 2}=1$ and therefore the correction is introduced in order to have the same upper critical point:

$$
b_{c 2}^{\text {corr }}=b_{c 2}^{*} / 0.985=1=b_{c 2} .
$$

This correction is dealt with in section 4.2. Moreover, Figure 10 shows the values of $F_{\min }$, (derived from Equations (16) (17) through the minimisation procedure) corresponding to the data in Annex. The box on each curve corresponds to the value of magnetic field $b_{3}$ (Equation (29)), and separates the areas of validity for Equations (25) (26) and (27) valid respectively to the left or to the right from the box for any $k>1$. Presented values of $F_{\min }$ can be used as a reference when comparing different methods of solving GL equations.

Finally, in order to illustrate the common feature of the ideal superconducting

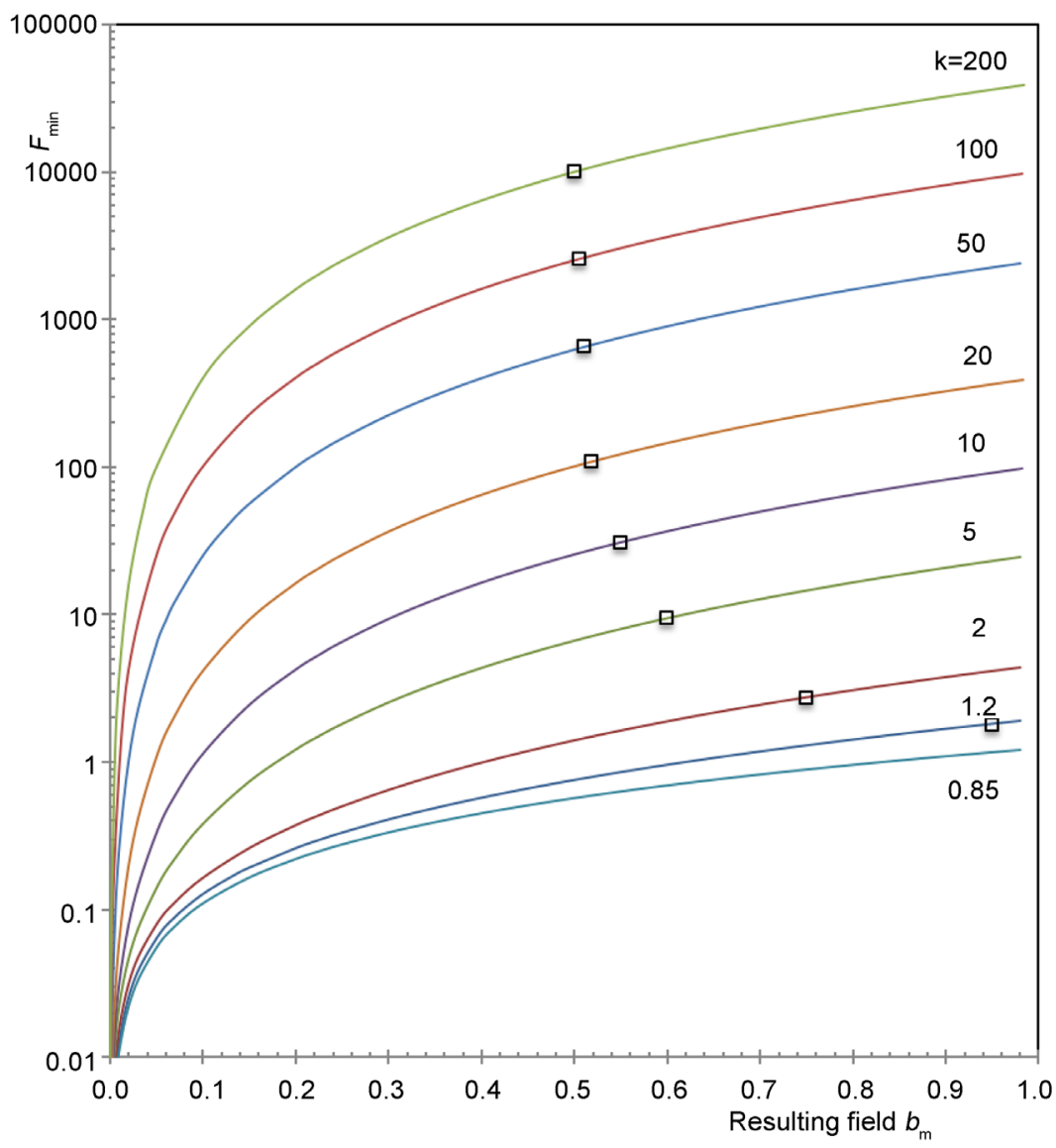

Figure 10. Minimum value $F_{\min }$ of the free energy density $F$ (Equation (16) (17)) as function of the resulting magnetic field $b_{m}$ for the selected values of $k$ (shown next to the corresponding lines). 
materials (in this case homogeneous, bulk, elliptically shaped, edge- and pin-free, placed in uniform magnetic field), in Figure 11 I show the magnetisation calculated for selected values of $k=34 ; 50$ and 75 (typical for $\mathrm{Nb}_{3} \mathrm{Sn}$; $\mathrm{NbTi}$ and REBCO respectively) and assuming vortex lattice with hexagonal unit cells. The solid lines represent $m_{1}$ as proposed here; the dashed line $-m_{3}$ (interpolated from the fit [[3], Equation (19)]). The scaling factor $m_{40}$ for the magnetisation in all cases is derived from Equation (28) (assuming $b_{a}=0$ ), in addition to that the scaling factor of 6 is used in order to keep the magnetisation values below 1 in the figure. Scaled this way magnetic field dependence of the magnetisation for these very different materials in the field range $0.001 \leq \bar{b} \leq 1$ collapse practically at the same curve. At lower magnetic fields there is a stratification depending on the $k$ value, besides $m_{3}$ gives errors as expected [3] that are higher at lower $k$.

\section{Conclusion}

Known symbolic method [5] [6] [8] [9] [10] of solving Ginzburg-Landau equations has limited validity. Namely, assumed dependencies (interpolation fits) for the variational parameters $f_{\infty}$ and $\xi_{v}$ on the Ginzburg-Landau parameter $k$

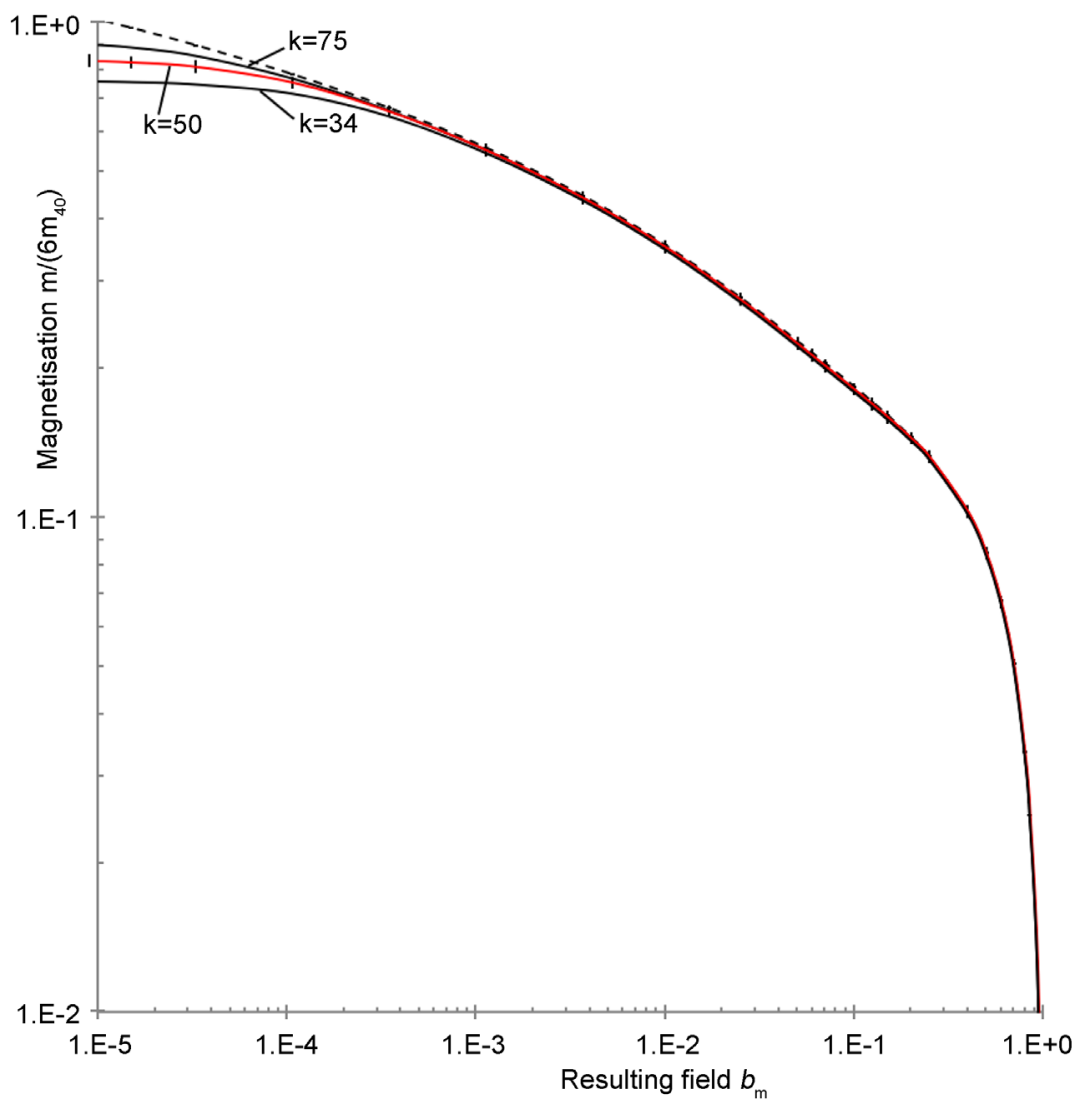

Figure 11. Calculated for $k=34,50$ and 75 magnetisation as function of the field $\bar{b}=b_{m}$ : the solid lines $-m_{1}$ as proposed here; the dashed line $-m_{3}$ (interpolated from the fit [[3], Equation (19)]). The scaling factor for the magnetisation in all cases is derived from Equation (28), in addition to that the scaling factor of 6 is used in order to keep the magnetisation ranging from 0.01 to 1 in the figure. The $\pm 3 \%$ relative error (the vertical bars) is guide for an eye. 
and on magnetic flux density $\bar{b}$ are inaccurate and result in unacceptably high errors (reaching 25\% and more). This limits applicability of otherwise excellent method. In the paper I eliminate several errors, extract and combine accurate symbolic solutions from the above and from [7] and provide for the first time precise dependencies for $f_{\infty}$ and $\xi_{v}$ on $k$ and $\bar{b}$ together with the simple and validated way of minimising the free energy density of superconductor. Resulting good agreement ( $98.5 \%$ for the entire range of magnetic flux density: $0.01 \leq \bar{b} \leq 1$ and any value of the parameter $k>1 / \sqrt{2}$ ) between the advanced symbolic and the known numerical solutions of Ginzburg-Landau equations validates both and hopefully will result in wider use of the symbolic approach.

\section{Acknowledgements}

If you are interested to support this study, contact the author at: o.a.chevtchenko@gmail.com. If you are interested in the results, contact us at: http://www.hts-powercables.nl. This research is funded privately and all rights belong to the author. Many thanks to A. A. Shevchenko for helping with difficult parts of this study, to R. Bakker for the inspirational atmosphere, to prof.-em. J. J. Smit for his support of this approach. A word of disgrace goes personally to Mr Jose Labastida, head of scientific department and to prof.-em. Helga Nowotny, former president of ERC, who chose to deny a funding for this study and thus created obstacles on the way to this result that could otherwise be published years earlier.

\section{References}

[1] Ginzburg, V.L. and Landau, L.D. (1950) Towards the Superconductivity Theory. Journal of Experimental and Theoretical Physics, 20, 1064.

[2] Bardeen, J., Cooper, L.N. and Schrieffer, J.R. (1957) Microscopic Theory of Superconductivity. Physical Review, 106, 162.

[3] Brandt, E.H. (2003) Properties of the Ideal Ginzburg-Landau Vortex Lattice. Physical Review B, 68, Article ID: 054506.

[4] Brandt, E.H. (2012) Mixed State Properties. In: Rogalla, H. and Kees, P., Eds., 100 Years of Superconductivity, Taylor \& Francis Group, Abingdon-on-Thames, 125137.

[5] Clem, J.R. (1975) Simple Model for the Vortex Core in a Type II Superconductor. Journal of Low Temperature Physics, 18, 427.

[6] Hao, Z. (1991) Model for the Reversible Magnetization of High-[Kappa] Type-II Superconductors: Application to High-Temperature Superconductors. PhD Book, Iowa State University, Ames.

[7] Abrikosov, A.A. (1957) On the Magnetic Properties of Superconductors of the Second Group. Journal of Experimental and Theoretical Physics, 5, 1174.

[8] Pogosov, V.V., Rakhmanov, A.L. and Kugel, K.I. (2000) Magnetization of Type-II Superconductors. Journal of Experimental and Theoretical Physics, 19, 588.

[9] Pogosov, V.V., Kugel, K.I., Rakhmanov, A.L. and Brandt, E.H. (2001) Approximate Ginzburg-Landau Solution for the Regular Flux-Line Lattice. Circular Cell Method. Physical Review B, 64, Article ID: 064517.

[10] Pogosov, V.V. (2002) Влияние поверхности и размеров образца на структуру 
смешанного состояния сверхпроводников второго рода. PhD Book, Moscow. (In Russian)

[11] Chevtchenko, O.A., Smit, J.J., de Vries, D.J. and de Pont, F.W.A. (2014) Modelling of Magnetisation and Intrinsic Properties of Ideal Type II Superconductor in External Magnetic Field. Proceedings of the 2014 COMSOL Conference, Cambridge. https://www.comsol.com/paper/download/199917/chevtchenko_paper.pdf

[12] Alstrøm, T.S., Sørensen, M.P., Pedersen, N.F. and Madsen, S. (2011) Magnetic Flux Lines in Complex Geometry Type-II Superconductors Studied by the Time Dependent Ginzburg-Landau Equation. Acta Applied Mathematics, 115, 63. https://doi.org/10.1007/s10440-010-9580-8

[13] Saint-James, D., Sarma, G. and Thomas, E.J. (1969) Type II Superconductivity. Elsevier Science \& Technology. 


\section{Annex}

Accurate dependencies of the variational parameters $\xi_{v}(k, \bar{b})$ and $f_{\infty}(k, \bar{b})$ obtained from the minimising the free energy density F, see Equations (16) (17) (Tables A1-A4). The dimensional and dimensionless quantities and scaling factors (Table A5).

Note that in order to simplify comparisons with [8] [9] [10] in Tables A1-A4 the values of $\bar{b}$ are not corrected (by 0.985 ).

Table A1. Spline data for the calculated variational parameter $f_{\infty}$ at $0.75 \leq k \leq 200$.

\begin{tabular}{|c|c|c|c|c|c|c|c|c|c|}
\hline \multicolumn{2}{|c|}{$k=0.75$} & \multicolumn{2}{|c|}{$k=0.85$} & \multicolumn{2}{|c|}{$k=1.2$} & \multicolumn{2}{|c|}{$k=2$} & \multicolumn{2}{|c|}{$5 \leq k \leq 200$} \\
\hline $\bar{b}$ & $f_{\infty}$ & $\bar{b}$ & $f_{\infty}$ & $\bar{b}$ & $f_{\infty}$ & $\bar{b}$ & $f_{\infty}$ & $\bar{b}$ & $f_{\infty}$ \\
\hline $7.26 \mathrm{E}-6$ & $1.000 \mathrm{E}+0$ & $5.95 \mathrm{E}-6$ & $1.000 \mathrm{E}+0$ & $2.96 \mathrm{E}-6$ & $1.000 \mathrm{E}+0$ & $1.05 \mathrm{E}-6$ & $1.000 \mathrm{E}+0$ & $5.80 \mathrm{E}-10$ & $1.000 \mathrm{E}+0$ \\
\hline $3.46 \mathrm{E}-4$ & $1.001 \mathrm{E}+0$ & $3.46 \mathrm{E}-4$ & $1.001 \mathrm{E}+0$ & $3.46 \mathrm{E}-4$ & $1.001 \mathrm{E}+0$ & $3.46 \mathrm{E}-4$ & $1.001 \mathrm{E}+0$ & $3.456 \mathrm{E}-4$ & $1.001 \mathrm{E}+0$ \\
\hline $1.12 \mathrm{E}-3$ & $1.001 \mathrm{E}+0$ & $1.12 \mathrm{E}-3$ & $1.001 \mathrm{E}+0$ & $1.12 \mathrm{E}-3$ & $1.002 \mathrm{E}+0$ & $1.12 \mathrm{E}-3$ & $1.002 \mathrm{E}+0$ & $1.121 \mathrm{E}-3$ & $1.002 \mathrm{E}+0$ \\
\hline $1.00 \mathrm{E}-2$ & $1.008 \mathrm{E}+0$ & $1.00 \mathrm{E}-2$ & $1.009 \mathrm{E}+0$ & $1.00 \mathrm{E}-2$ & $1.010 \mathrm{E}+0$ & $3.68 \mathrm{E}-3$ & $1.005 \mathrm{E}+0$ & $1.287 \mathrm{E}-2$ & $1.014 \mathrm{E}+0$ \\
\hline $2.50 \mathrm{E}-2$ & $1.017 \mathrm{E}+0$ & $2.50 \mathrm{E}-2$ & $1.018 \mathrm{E}+0$ & $2.50 \mathrm{E}-2$ & $1.020 \mathrm{E}+0$ & $7.00 \mathrm{E}-3$ & $1.008 \mathrm{E}+0$ & $2.500 \mathrm{E}-2$ & $1.024 \mathrm{E}+0$ \\
\hline $5.00 \mathrm{E}-2$ & $1.029 \mathrm{E}+0$ & $5.00 \mathrm{E}-2$ & $1.030 \mathrm{E}+0$ & $5.00 \mathrm{E}-2$ & $1.034 \mathrm{E}+0$ & $1.00 \mathrm{E}-2$ & $1.011 \mathrm{E}+0$ & $5.000 \mathrm{E}-2$ & $1.042 \mathrm{E}+0$ \\
\hline $7.00 \mathrm{E}-2$ & $1.036 \mathrm{E}+0$ & $7.00 \mathrm{E}-2$ & $1.038 \mathrm{E}+0$ & $7.00 \mathrm{E}-2$ & $1.043 \mathrm{E}+0$ & $2.00 \mathrm{E}-2$ & $1.019 \mathrm{E}+0$ & $7.000 \mathrm{E}-2$ & $1.053 \mathrm{E}+0$ \\
\hline $1.00 \mathrm{E}-1$ & $1.046 \mathrm{E}+0$ & $1.00 \mathrm{E}-1$ & $1.048 \mathrm{E}+0$ & $1.00 \mathrm{E}-1$ & $1.054 \mathrm{E}+0$ & $3.50 \mathrm{E}-2$ & $1.029 \mathrm{E}+0$ & $1.000 \mathrm{E}-1$ & $1.065 \mathrm{E}+0$ \\
\hline $2.00 \mathrm{E}-1$ & $1.058 \mathrm{E}+0$ & $2.00 \mathrm{E}-1$ & $1.060 \mathrm{E}+0$ & $2.00 \mathrm{E}-1$ & $1.065 \mathrm{E}+0$ & $7.00 \mathrm{E}-2$ & $1.048 \mathrm{E}+0$ & $2.000 \mathrm{E}-1$ & $1.071 \mathrm{E}+0$ \\
\hline $2.25 \mathrm{E}-1$ & $1.056 \mathrm{E}+0$ & $2.25 \mathrm{E}-1$ & $1.058 \mathrm{E}+0$ & $2.25 \mathrm{E}-1$ & $1.062 \mathrm{E}+0$ & $1.00 \mathrm{E}-1$ & $1.060 \mathrm{E}+0$ & $2.250 \mathrm{E}-1$ & $1.066 \mathrm{E}+0$ \\
\hline $2.50 \mathrm{E}-1$ & $1.053 \mathrm{E}+0$ & $2.50 \mathrm{E}-1$ & $1.055 \mathrm{E}+0$ & $2.50 \mathrm{E}-1$ & $1.057 \mathrm{E}+0$ & $1.50 \mathrm{E}-1$ & $1.070 \mathrm{E}+0$ & $2.500 \mathrm{E}-1$ & $1.059 \mathrm{E}+0$ \\
\hline $3.00 \mathrm{E}-1$ & $1.042 \mathrm{E}+0$ & $3.00 \mathrm{E}-1$ & $1.042 \mathrm{E}+0$ & $3.00 \mathrm{E}-1$ & $1.042 \mathrm{E}+0$ & $2.00 \mathrm{E}-1$ & $1.068 \mathrm{E}+0$ & $3.000 \mathrm{E}-1$ & $1.040 \mathrm{E}+0$ \\
\hline $3.50 \mathrm{E}-1$ & $1.025 \mathrm{E}+0$ & $3.50 \mathrm{E}-1$ & $1.024 \mathrm{E}+0$ & $3.50 \mathrm{E}-1$ & $1.021 \mathrm{E}+0$ & $2.25 \mathrm{E}-1$ & $1.064 \mathrm{E}+0$ & $3.500 \mathrm{E}-1$ & $1.016 \mathrm{E}+0$ \\
\hline $4.00 \mathrm{E}-1$ & $1.002 \mathrm{E}+0$ & $4.00 \mathrm{E}-1$ & $9.996 \mathrm{E}-1$ & $4.00 \mathrm{E}-1$ & $9.939 \mathrm{E}-1$ & $2.50 \mathrm{E}-1$ & $1.058 \mathrm{E}+0$ & $4.000 \mathrm{E}-1$ & $9.860 \mathrm{E}-1$ \\
\hline $4.50 \mathrm{E}-1$ & $9.738 \mathrm{E}-1$ & $4.50 \mathrm{E}-1$ & $9.701 \mathrm{E}-1$ & $4.50 \mathrm{E}-1$ & $9.621 \mathrm{E}-1$ & $3.00 \mathrm{E}-1$ & $1.041 \mathrm{E}+0$ & $4.500 \mathrm{E}-1$ & $9.519 \mathrm{E}-1$ \\
\hline $5.00 \mathrm{E}-1$ & $9.404 \mathrm{E}-1$ & $5.00 \mathrm{E}-1$ & $9.355 \mathrm{E}-1$ & $5.00 \mathrm{E}-1$ & $9.255 \mathrm{E}-1$ & $3.50 \mathrm{E}-1$ & $1.018 \mathrm{E}+0$ & $5.000 \mathrm{E}-1$ & $9.136 \mathrm{E}-1$ \\
\hline $5.50 \mathrm{E}-1$ & $9.018 \mathrm{E}-1$ & $5.50 \mathrm{E}-1$ & $8.959 \mathrm{E}-1$ & $5.50 \mathrm{E}-1$ & $8.843 \mathrm{E}-1$ & $4.00 \mathrm{E}-1$ & $9.891 \mathrm{E}-1$ & $5.500 \mathrm{E}-1$ & $8.712 \mathrm{E}-1$ \\
\hline $6.00 \mathrm{E}-1$ & $8.579 \mathrm{E}-1$ & $6.00 \mathrm{E}-1$ & $8.511 \mathrm{E}-1$ & $6.00 \mathrm{E}-1$ & $8.384 \mathrm{E}-1$ & $4.50 \mathrm{E}-1$ & $9.558 \mathrm{E}-1$ & $6.000 \mathrm{E}-1$ & $8.245 \mathrm{E}-1$ \\
\hline $7.50 \mathrm{E}-1$ & $6.884 \mathrm{E}-1$ & $7.50 \mathrm{E}-1$ & $6.806 \mathrm{E}-1$ & $7.50 \mathrm{E}-1$ & $6.668 \mathrm{E}-1$ & $6.00 \mathrm{E}-1$ & $8.296 \mathrm{E}-1$ & $7.500 \mathrm{E}-1$ & $6.529 \mathrm{E}-1$ \\
\hline $8.00 \mathrm{E}-1$ & $6.152 \mathrm{E}-1$ & $8.00 \mathrm{E}-1$ & $6.075 \mathrm{E}-1$ & $8.00 \mathrm{E}-1$ & $5.943 \mathrm{E}-1$ & $6.50 \mathrm{E}-1$ & $7.782 \mathrm{E}-1$ & $8.000 \mathrm{E}-1$ & $5.812 \mathrm{E}-1$ \\
\hline $8.50 \mathrm{E}-1$ & $5.288 \mathrm{E}-1$ & $8.50 \mathrm{E}-1$ & $5.217 \mathrm{E}-1$ & $8.50 \mathrm{E}-1$ & $5.095 \mathrm{E}-1$ & $7.00 \mathrm{E}-1$ & $7.214 \mathrm{E}-1$ & $8.500 \mathrm{E}-1$ & $4.978 \mathrm{E}-1$ \\
\hline $9.00 \mathrm{E}-1$ & $4.217 \mathrm{E}-1$ & $9.00 \mathrm{E}-1$ & $4.156 \mathrm{E}-1$ & $9.00 \mathrm{E}-1$ & $4.054 \mathrm{E}-1$ & $7.50 \mathrm{E}-1$ & $6.579 \mathrm{E}-1$ & $9.000 \mathrm{E}-1$ & $3.957 \mathrm{E}-1$ \\
\hline $9.50 \mathrm{E}-1$ & $2.708 \mathrm{E}-1$ & $9.50 \mathrm{E}-1$ & $2.667 \mathrm{E}-1$ & $9.50 \mathrm{E}-1$ & $2.598 \mathrm{E}-1$ & $8.00 \mathrm{E}-1$ & $5.858 \mathrm{E}-1$ & $9.500 \mathrm{E}-1$ & $2.534 \mathrm{E}-1$ \\
\hline $9.75 \mathrm{E}-1$ & $1.421 \mathrm{E}-1$ & $9.75 \mathrm{E}-1$ & $1.398 \mathrm{E}-1$ & $9.75 \mathrm{E}-1$ & $1.362 \mathrm{E}-1$ & $8.50 \mathrm{E}-1$ & $5.020 \mathrm{E}-1$ & $9.750 \mathrm{E}-1$ & $1.327 \mathrm{E}-1$ \\
\hline $9.80 \mathrm{E}-1$ & $9.740 \mathrm{E}-2$ & $9.80 \mathrm{E}-1$ & $9.586 \mathrm{E}-2$ & $9.80 \mathrm{E}-1$ & $9.333 \mathrm{E}-2$ & $9.00 \mathrm{E}-1$ & $3.991 \mathrm{E}-1$ & $9.800 \mathrm{E}-1$ & $9.099 \mathrm{E}-2$ \\
\hline \multirow[t]{5}{*}{$9.85 \mathrm{E}-1$} & $1.000 \mathrm{E}-4$ & $9.85 \mathrm{E}-1$ & $1.000 \mathrm{E}-4$ & $9.85 \mathrm{E}-1$ & $1.000 \mathrm{E}-4$ & $9.50 \mathrm{E}-1$ & $2.556 \mathrm{E}-1$ & $9.825 \mathrm{E}-1$ & $5.995 \mathrm{E}-2$ \\
\hline & & & & & & $9.60 \mathrm{E}-1$ & $2.154 \mathrm{E}-1$ & $9.830 \mathrm{E}-1$ & $5.162 \mathrm{E}-2$ \\
\hline & & & & & & $9.70 \mathrm{E}-1$ & $1.656 \mathrm{E}-1$ & $9.850 \mathrm{E}-1$ & $1.000 \mathrm{E}-4$ \\
\hline & & & & & & $9.80 \mathrm{E}-1$ & $9.180 \mathrm{E}-2$ & & \\
\hline & & & & & & $9.85 \mathrm{E}-1$ & $1.000 \mathrm{E}-4$ & & \\
\hline
\end{tabular}


Table A2. Spline data for the calculated variational parameter $\xi_{v} / \xi_{v 0}$ at $k \geq 50$.

\begin{tabular}{|c|c|c|c|c|c|}
\hline \multicolumn{2}{|c|}{$k=50$} & \multicolumn{2}{|c|}{$k=100$} & \multicolumn{2}{|c|}{$k=200$} \\
\hline $\bar{b}$ & $\xi_{v} / \xi_{v 0}$ & $\bar{b}$ & $\xi_{v} / \xi_{v 0}$ & $\bar{b}$ & $\xi_{v} / \xi_{v 0}$ \\
\hline $7.025 \mathrm{E}-9$ & $9.989 \mathrm{E}-1$ & $9.090 \mathrm{E}-6$ & $1.000 \mathrm{E}+0$ & $1.318 \mathrm{E}-10$ & $1.000 \mathrm{E}+0$ \\
\hline $9.000 \mathrm{E}-6$ & $1.001 \mathrm{E}+0$ & $3.287 \mathrm{E}-5$ & $1.002 \mathrm{E}+0$ & $1.000 \mathrm{E}-6$ & $1.000 \mathrm{E}+0$ \\
\hline $1.500 \mathrm{E}-5$ & $1.001 \mathrm{E}+0$ & $1.073 \mathrm{E}-4$ & $1.003 \mathrm{E}+0$ & $9.090 \mathrm{E}-6$ & $1.001 \mathrm{E}+0$ \\
\hline $3.287 \mathrm{E}-5$ & $1.002 \mathrm{E}+0$ & $3.456 \mathrm{E}-4$ & $1.007 \mathrm{E}+0$ & $3.287 \mathrm{E}-5$ & $1.001 \mathrm{E}+0$ \\
\hline $1.073 \mathrm{E}-4$ & $1.004 \mathrm{E}+0$ & $1.121 \mathrm{E}-3$ & $1.014 \mathrm{E}+0$ & $1.073 \mathrm{E}-4$ & $1.003 \mathrm{E}+0$ \\
\hline $3.456 \mathrm{E}-4$ & $1.008 \mathrm{E}+0$ & $3.682 \mathrm{E}-3$ & $1.029 \mathrm{E}+0$ & $3.456 \mathrm{E}-4$ & $1.006 \mathrm{E}+0$ \\
\hline $1.121 \mathrm{E}-3$ & $1.016 \mathrm{E}+0$ & $1.287 \mathrm{E}-2$ & $1.056 \mathrm{E}+0$ & $1.121 \mathrm{E}-3$ & $1.014 \mathrm{E}+0$ \\
\hline $3.682 \mathrm{E}-3$ & $1.031 \mathrm{E}+0$ & $2.500 \mathrm{E}-2$ & $1.075 \mathrm{E}+0$ & $5.000 \mathrm{E}-3$ & $1.033 \mathrm{E}+0$ \\
\hline $1.000 \mathrm{E}-2$ & $1.052 \mathrm{E}+0$ & $5.000 \mathrm{E}-2$ & $1.095 \mathrm{E}+0$ & $2.000 \mathrm{E}-2$ & $1.067 \mathrm{E}+0$ \\
\hline $2.500 \mathrm{E}-2$ & $1.079 \mathrm{E}+0$ & $7.000 \mathrm{E}-2$ & $1.099 \mathrm{E}+0$ & $5.000 \mathrm{E}-2$ & $1.093 \mathrm{E}+0$ \\
\hline $5.000 \mathrm{E}-2$ & $1.098 \mathrm{E}+0$ & $1.000 \mathrm{E}-1$ & $1.095 \mathrm{E}+0$ & $1.000 \mathrm{E}-1$ & $1.094 \mathrm{E}+0$ \\
\hline $7.000 \mathrm{E}-2$ & $1.103 \mathrm{E}+0$ & $1.500 \mathrm{E}-1$ & $1.072 \mathrm{E}+0$ & $1.500 \mathrm{E}-1$ & $1.071 \mathrm{E}+0$ \\
\hline $1.000 \mathrm{E}-1$ & $1.099 \mathrm{E}+0$ & $2.000 \mathrm{E}-1$ & $1.041 \mathrm{E}+0$ & $2.000 \mathrm{E}-1$ & $1.040 \mathrm{E}+0$ \\
\hline $1.500 \mathrm{E}-1$ & $1.076 \mathrm{E}+0$ & $2.250 \mathrm{E}-1$ & $1.024 \mathrm{E}+0$ & $2.250 \mathrm{E}-1$ & $1.023 \mathrm{E}+0$ \\
\hline $2.000 \mathrm{E}-1$ & $1.044 \mathrm{E}+0$ & $2.500 \mathrm{E}-1$ & $1.006 \mathrm{E}+0$ & $2.500 \mathrm{E}-1$ & $1.006 \mathrm{E}+0$ \\
\hline $2.250 \mathrm{E}-1$ & $1.027 \mathrm{E}+0$ & $3.000 \mathrm{E}-1$ & $9.725 \mathrm{E}-1$ & $3.000 \mathrm{E}-1$ & $9.718 \mathrm{E}-1$ \\
\hline $2.500 \mathrm{E}-1$ & $1.010 \mathrm{E}+0$ & $3.500 \mathrm{E}-1$ & $9.402 \mathrm{E}-1$ & $3.500 \mathrm{E}-1$ & $9.395 \mathrm{E}-1$ \\
\hline $3.000 \mathrm{E}-1$ & $9.759 \mathrm{E}-1$ & $4.000 \mathrm{E}-1$ & $9.098 \mathrm{E}-1$ & $4.000 \mathrm{E}-1$ & $9.092 \mathrm{E}-1$ \\
\hline $3.500 \mathrm{E}-1$ & $9.435 \mathrm{E}-1$ & $4.500 \mathrm{E}-1$ & $8.816 \mathrm{E}-1$ & $4.500 \mathrm{E}-1$ & $8.810 \mathrm{E}-1$ \\
\hline $4.000 \mathrm{E}-1$ & $9.130 \mathrm{E}-1$ & $5.000 \mathrm{E}-1$ & $8.553 \mathrm{E}-1$ & $5.000 \mathrm{E}-1$ & $8.548 \mathrm{E}-1$ \\
\hline $4.500 \mathrm{E}-1$ & $8.847 \mathrm{E}-1$ & $5.500 \mathrm{E}-1$ & $8.310 \mathrm{E}-1$ & $5.500 \mathrm{E}-1$ & $8.305 \mathrm{E}-1$ \\
\hline $5.000 \mathrm{E}-1$ & $8.583 \mathrm{E}-1$ & $6.000 \mathrm{E}-1$ & $8.084 \mathrm{E}-1$ & $6.000 \mathrm{E}-1$ & $8.079 \mathrm{E}-1$ \\
\hline $5.500 \mathrm{E}-1$ & $8.339 \mathrm{E}-1$ & $6.500 \mathrm{E}-1$ & $7.875 \mathrm{E}-1$ & $6.500 \mathrm{E}-1$ & $7.869 \mathrm{E}-1$ \\
\hline $6.000 \mathrm{E}-1$ & $8.113 \mathrm{E}-1$ & $7.000 \mathrm{E}-1$ & $7.679 \mathrm{E}-1$ & $7.000 \mathrm{E}-1$ & $7.674 \mathrm{E}-1$ \\
\hline $6.500 \mathrm{E}-1$ & $7.902 \mathrm{E}-1$ & $7.500 \mathrm{E}-1$ & $7.497 \mathrm{E}-1$ & $7.500 \mathrm{E}-1$ & $7.492 \mathrm{E}-1$ \\
\hline $7.000 \mathrm{E}-1$ & $7.706 \mathrm{E}-1$ & $8.000 \mathrm{E}-1$ & $7.326 \mathrm{E}-1$ & $8.000 \mathrm{E}-1$ & $7.321 \mathrm{E}-1$ \\
\hline $7.500 \mathrm{E}-1$ & $7.523 \mathrm{E}-1$ & $8.500 \mathrm{E}-1$ & $7.166 \mathrm{E}-1$ & $8.500 \mathrm{E}-1$ & $7.161 \mathrm{E}-1$ \\
\hline $8.000 \mathrm{E}-1$ & $7.352 \mathrm{E}-1$ & $9.000 \mathrm{E}-1$ & $7.015 \mathrm{E}-1$ & $9.000 \mathrm{E}-1$ & $7.010 \mathrm{E}-1$ \\
\hline $8.500 \mathrm{E}-1$ & $7.191 \mathrm{E}-1$ & $9.500 \mathrm{E}-1$ & $6.874 \mathrm{E}-1$ & $9.400 \mathrm{E}-1$ & $6.893 \mathrm{E}-1$ \\
\hline $9.000 \mathrm{E}-1$ & $7.040 \mathrm{E}-1$ & $9.750 \mathrm{E}-1$ & $6.805 \mathrm{E}-1$ & $9.500 \mathrm{E}-1$ & $6.869 \mathrm{E}-1$ \\
\hline $9.500 \mathrm{E}-1$ & $6.898 \mathrm{E}-1$ & $9.800 \mathrm{E}-1$ & $6.792 \mathrm{E}-1$ & $9.600 \mathrm{E}-1$ & $6.837 \mathrm{E}-1$ \\
\hline $9.750 \mathrm{E}-1$ & $6.829 \mathrm{E}-1$ & $9.825 \mathrm{E}-1$ & $6.785 \mathrm{E}-1$ & $9.700 \mathrm{E}-1$ & $6.815 \mathrm{E}-1$ \\
\hline $9.800 \mathrm{E}-1$ & $6.817 \mathrm{E}-1$ & $9.830 \mathrm{E}-1$ & $6.784 \mathrm{E}-1$ & $9.790 \mathrm{E}-1$ & $6.786 \mathrm{E}-1$ \\
\hline $9.850 \mathrm{E}-1$ & $6.817 \mathrm{E}-1$ & $9.850 \mathrm{E}-1$ & $6.784 \mathrm{E}-1$ & $9.840 \mathrm{E}-1$ & $6.774 \mathrm{E}-1$ \\
\hline
\end{tabular}


Table A3. Spline data for the calculated variational parameter $\xi_{v} / \xi_{v 0}$ at $0.75<k \leq 2$.

\begin{tabular}{|c|c|c|c|c|c|c|c|}
\hline \multicolumn{2}{|c|}{$k=0.75$} & \multicolumn{2}{|c|}{$k=0.85$} & \multicolumn{2}{|c|}{$k=1.2$} & \multicolumn{2}{|c|}{$k=2$} \\
\hline $\bar{b}$ & $\xi_{v} / \xi_{v 0}$ & $\bar{b}$ & $\xi_{v} / \xi_{v 0}$ & $\bar{b}$ & $\xi_{v} / \xi_{v 0}$ & $\bar{b}$ & $\xi_{v} / \xi_{v 0}$ \\
\hline $7.258 \mathrm{E}-6$ & $1.001 \mathrm{E}+0$ & $5.954 \mathrm{E}-6$ & $9.946 \mathrm{E}-1$ & $2.962 \mathrm{E}-6$ & $1.000 \mathrm{E}+0$ & $3.287 \mathrm{E}-5$ & $1.001 \mathrm{E}+0$ \\
\hline $9.000 \mathrm{E}-6$ & $1.001 \mathrm{E}+0$ & $7.000 \mathrm{E}-6$ & $9.946 \mathrm{E}-1$ & $9.000 \mathrm{E}-6$ & $1.000 \mathrm{E}+0$ & $1.073 \mathrm{E}-4$ & $1.001 \mathrm{E}+0$ \\
\hline $1.500 \mathrm{E}-5$ & $1.001 \mathrm{E}+0$ & $9.000 \mathrm{E}-6$ & $9.946 \mathrm{E}-1$ & $1.000 \mathrm{E}-5$ & $1.000 \mathrm{E}+0$ & $3.456 \mathrm{E}-4$ & $1.008 \mathrm{E}+0$ \\
\hline $3.287 \mathrm{E}-5$ & $1.001 \mathrm{E}+0$ & $3.287 \mathrm{E}-5$ & $9.946 \mathrm{E}-1$ & $3.287 \mathrm{E}-5$ & $1.000 \mathrm{E}+0$ & $1.121 \mathrm{E}-3$ & $1.019 \mathrm{E}+0$ \\
\hline $1.073 \mathrm{E}-4$ & $1.001 \mathrm{E}+0$ & $1.073 \mathrm{E}-4$ & $9.946 \mathrm{E}-1$ & $1.073 \mathrm{E}-4$ & $1.000 \mathrm{E}+0$ & $2.000 \mathrm{E}-3$ & $1.027 \mathrm{E}+0$ \\
\hline $3.456 \mathrm{E}-4$ & $1.006 \mathrm{E}+0$ & $3.456 \mathrm{E}-4$ & $1.006 \mathrm{E}+0$ & $3.456 \mathrm{E}-4$ & $1.007 \mathrm{E}+0$ & $3.682 \mathrm{E}-3$ & $1.041 \mathrm{E}+0$ \\
\hline $1.121 \mathrm{E}-3$ & $1.013 \mathrm{E}+0$ & $1.121 \mathrm{E}-3$ & $1.014 \mathrm{E}+0$ & $1.121 \mathrm{E}-3$ & $1.016 \mathrm{E}+0$ & $7.000 \mathrm{E}-3$ & $1.061 \mathrm{E}+0$ \\
\hline $4.000 \mathrm{E}-3$ & $1.031 \mathrm{E}+0$ & $3.682 \mathrm{E}-3$ & $1.031 \mathrm{E}+0$ & $3.682 \mathrm{E}-3$ & $1.035 \mathrm{E}+0$ & $1.000 \mathrm{E}-2$ & $1.076 \mathrm{E}+0$ \\
\hline $1.000 \mathrm{E}-2$ & $1.056 \mathrm{E}+0$ & $1.000 \mathrm{E}-2$ & $1.059 \mathrm{E}+0$ & $1.000 \mathrm{E}-2$ & $1.066 \mathrm{E}+0$ & $2.000 \mathrm{E}-2$ & $1.116 \mathrm{E}+0$ \\
\hline $2.500 \mathrm{E}-2$ & $1.097 \mathrm{E}+0$ & $2.500 \mathrm{E}-2$ & $1.102 \mathrm{E}+0$ & $2.500 \mathrm{E}-2$ & $1.115 \mathrm{E}+0$ & $3.500 \mathrm{E}-2$ & $1.157 \mathrm{E}+0$ \\
\hline $5.000 \mathrm{E}-2$ & $1.142 \mathrm{E}+0$ & $5.000 \mathrm{E}-2$ & $1.149 \mathrm{E}+0$ & $5.000 \mathrm{E}-2$ & $1.168 \mathrm{E}+0$ & $5.000 \mathrm{E}-2$ & $1.188 \mathrm{E}+0$ \\
\hline $7.000 \mathrm{E}-2$ & $1.168 \mathrm{E}+0$ & $7.000 \mathrm{E}-2$ & $1.177 \mathrm{E}+0$ & $7.000 \mathrm{E}-2$ & $1.198 \mathrm{E}+0$ & $7.000 \mathrm{E}-2$ & $1.215 \mathrm{E}+0$ \\
\hline $1.000 \mathrm{E}-1$ & $1.197 \mathrm{E}+0$ & $1.000 \mathrm{E}-1$ & $1.206 \mathrm{E}+0$ & $1.000 \mathrm{E}-1$ & $1.229 \mathrm{E}+0$ & $1.000 \mathrm{E}-1$ & $1.237 \mathrm{E}+0$ \\
\hline $1.500 \mathrm{E}-1$ & $1.225 \mathrm{E}+0$ & $1.500 \mathrm{E}-1$ & $1.234 \mathrm{E}+0$ & $1.337 \mathrm{E}-1$ & $1.247 \mathrm{E}+0$ & $1.500 \mathrm{E}-1$ & $1.242 \mathrm{E}+0$ \\
\hline $2.000 \mathrm{E}-1$ & $1.236 \mathrm{E}+0$ & $2.000 \mathrm{E}-1$ & $1.244 \mathrm{E}+0$ & $1.500 \mathrm{E}-1$ & $1.252 \mathrm{E}+0$ & $2.000 \mathrm{E}-1$ & $1.224 \mathrm{E}+0$ \\
\hline $2.250 \mathrm{E}-1$ & $1.237 \mathrm{E}+0$ & $2.250 \mathrm{E}-1$ & $1.244 \mathrm{E}+0$ & $2.000 \mathrm{E}-1$ & $1.252 \mathrm{E}+0$ & $2.250 \mathrm{E}-1$ & $1.211 \mathrm{E}+0$ \\
\hline $2.500 \mathrm{E}-1$ & $1.236 \mathrm{E}+0$ & $2.500 \mathrm{E}-1$ & $1.242 \mathrm{E}+0$ & $2.250 \mathrm{E}-1$ & $1.247 \mathrm{E}+0$ & $2.500 \mathrm{E}-1$ & $1.196 \mathrm{E}+0$ \\
\hline $3.000 \mathrm{E}-1$ & $1.230 \mathrm{E}+0$ & $3.000 \mathrm{E}-1$ & $1.231 \mathrm{E}+0$ & $2.500 \mathrm{E}-1$ & $1.239 \mathrm{E}+0$ & $3.000 \mathrm{E}-1$ & $1.165 \mathrm{E}+0$ \\
\hline $3.500 \mathrm{E}-1$ & $1.218 \mathrm{E}+0$ & $3.500 \mathrm{E}-1$ & $1.216 \mathrm{E}+0$ & $3.000 \mathrm{E}-1$ & $1.218 \mathrm{E}+0$ & $3.500 \mathrm{E}-1$ & $1.133 \mathrm{E}+0$ \\
\hline $4.000 \mathrm{E}-1$ & $1.203 \mathrm{E}+0$ & $4.000 \mathrm{E}-1$ & $1.198 \mathrm{E}+0$ & $3.500 \mathrm{E}-1$ & $1.194 \mathrm{E}+0$ & $4.000 \mathrm{E}-1$ & $1.101 \mathrm{E}+0$ \\
\hline $4.500 \mathrm{E}-1$ & $1.187 \mathrm{E}+0$ & $4.500 \mathrm{E}-1$ & $1.179 \mathrm{E}+0$ & $4.000 \mathrm{E}-1$ & $1.168 \mathrm{E}+0$ & $4.500 \mathrm{E}-1$ & $1.070 \mathrm{E}+0$ \\
\hline $5.000 \mathrm{E}-1$ & $1.170 \mathrm{E}+0$ & $5.000 \mathrm{E}-1$ & $1.159 \mathrm{E}+0$ & $4.500 \mathrm{E}-1$ & $1.141 \mathrm{E}+0$ & $5.000 \mathrm{E}-1$ & $1.041 \mathrm{E}+0$ \\
\hline $5.500 \mathrm{E}-1$ & $1.152 \mathrm{E}+0$ & $5.500 \mathrm{E}-1$ & $1.138 \mathrm{E}+0$ & $5.000 \mathrm{E}-1$ & $1.115 \mathrm{E}+0$ & $5.500 \mathrm{E}-1$ & $1.014 \mathrm{E}+0$ \\
\hline $6.000 \mathrm{E}-1$ & $1.134 \mathrm{E}+0$ & $6.000 \mathrm{E}-1$ & $1.118 \mathrm{E}+0$ & $5.500 \mathrm{E}-1$ & $1.090 \mathrm{E}+0$ & $6.000 \mathrm{E}-1$ & $9.885 \mathrm{E}-1$ \\
\hline $6.500 \mathrm{E}-1$ & $1.117 \mathrm{E}+0$ & $6.500 \mathrm{E}-1$ & $1.098 \mathrm{E}+0$ & $6.000 \mathrm{E}-1$ & $1.066 \mathrm{E}+0$ & $6.500 \mathrm{E}-1$ & $9.643 \mathrm{E}-1$ \\
\hline $7.000 \mathrm{E}-1$ & $1.099 \mathrm{E}+0$ & $7.000 \mathrm{E}-1$ & $1.079 \mathrm{E}+0$ & $6.500 \mathrm{E}-1$ & $1.043 \mathrm{E}+0$ & $7.000 \mathrm{E}-1$ & $9.417 \mathrm{E}-1$ \\
\hline $7.500 \mathrm{E}-1$ & $1.082 \mathrm{E}+0$ & $7.500 \mathrm{E}-1$ & $1.061 \mathrm{E}+0$ & $7.000 \mathrm{E}-1$ & $1.021 \mathrm{E}+0$ & $7.500 \mathrm{E}-1$ & $9.204 \mathrm{E}-1$ \\
\hline $8.000 \mathrm{E}-1$ & $1.066 \mathrm{E}+0$ & $8.000 \mathrm{E}-1$ & $1.043 \mathrm{E}+0$ & $7.500 \mathrm{E}-1$ & $1.000 \mathrm{E}+0$ & $8.000 \mathrm{E}-1$ & $9.004 \mathrm{E}-1$ \\
\hline $8.500 \mathrm{E}-1$ & $1.050 \mathrm{E}+0$ & $8.500 \mathrm{E}-1$ & $1.025 \mathrm{E}+0$ & $8.000 \mathrm{E}-1$ & $9.803 \mathrm{E}-1$ & $8.500 \mathrm{E}-1$ & $8.815 \mathrm{E}-1$ \\
\hline $9.000 \mathrm{E}-1$ & $1.035 \mathrm{E}+0$ & $9.000 \mathrm{E}-1$ & $1.009 \mathrm{E}+0$ & $8.500 \mathrm{E}-1$ & $9.614 \mathrm{E}-1$ & $9.000 \mathrm{E}-1$ & $8.637 \mathrm{E}-1$ \\
\hline $9.500 \mathrm{E}-1$ & $1.020 \mathrm{E}+0$ & $9.500 \mathrm{E}-1$ & $9.928 \mathrm{E}-1$ & $9.000 \mathrm{E}-1$ & $9.434 \mathrm{E}-1$ & $9.500 \mathrm{E}-1$ & $8.469 \mathrm{E}-1$ \\
\hline $9.750 \mathrm{E}-1$ & $1.012 \mathrm{E}+0$ & $9.750 \mathrm{E}-1$ & $9.850 \mathrm{E}-1$ & $9.500 \mathrm{E}-1$ & $9.263 \mathrm{E}-1$ & $9.600 \mathrm{E}-1$ & $8.437 \mathrm{E}-1$ \\
\hline $9.800 \mathrm{E}-1$ & $1.011 \mathrm{E}+0$ & $9.800 \mathrm{E}-1$ & $9.835 \mathrm{E}-1$ & $9.750 \mathrm{E}-1$ & $9.181 \mathrm{E}-1$ & $9.700 \mathrm{E}-1$ & $8.405 \mathrm{E}-1$ \\
\hline \multirow[t]{2}{*}{$9.850 \mathrm{E}-1$} & $1.011 \mathrm{E}+0$ & $9.850 \mathrm{E}-1$ & $9.835 \mathrm{E}-1$ & $9.800 \mathrm{E}-1$ & $9.165 \mathrm{E}-1$ & $9.800 \mathrm{E}-1$ & $8.373 \mathrm{E}-1$ \\
\hline & & & & $9.850 \mathrm{E}-1$ & $9.165 \mathrm{E}-1$ & $9.850 \mathrm{E}-1$ & $8.373 \mathrm{E}-1$ \\
\hline
\end{tabular}


Table A4. Spline data for the calculated variational parameter $\xi_{v} / \xi_{v 0}$ at $5 \leq k<50$.

\begin{tabular}{|c|c|c|c|c|c|c|c|}
\hline \multicolumn{2}{|c|}{$k=5$} & \multicolumn{2}{|c|}{$k=10$} & \multicolumn{2}{|c|}{$k=20$} & \multicolumn{2}{|c|}{$k=50^{*}$} \\
\hline $\bar{b}$ & $\xi_{v} / \xi_{v 0}$ & $\bar{b}$ & $\xi_{v} / \xi_{v 0}$ & $\bar{b}$ & $\xi_{v} / \xi_{v 0}$ & $\bar{b}$ & $\xi_{v} / \xi_{v 0}$ \\
\hline $2.000 \mathrm{E}-7$ & $1.001 \mathrm{E}+0$ & $4.000 \mathrm{E}-6$ & $1.000 \mathrm{E}+0$ & $1.000 \mathrm{E}-8$ & $1.001 \mathrm{E}+0$ & $7.025 \mathrm{E}-9$ & $9.989 \mathrm{E}-1$ \\
\hline $1.000 \mathrm{E}-6$ & $1.001 \mathrm{E}+0$ & $9.090 \mathrm{E}-6$ & $1.000 \mathrm{E}+0$ & $1.000 \mathrm{E}-6$ & $1.001 \mathrm{E}+0$ & $9.000 \mathrm{E}-6$ & $1.001 \mathrm{E}+0$ \\
\hline $9.090 \mathrm{E}-6$ & $1.001 \mathrm{E}+0$ & $3.287 \mathrm{E}-5$ & $1.000 \mathrm{E}+0$ & $9.090 \mathrm{E}-6$ & $1.001 \mathrm{E}+0$ & $1.500 \mathrm{E}-5$ & $1.001 \mathrm{E}+0$ \\
\hline $3.287 \mathrm{E}-5$ & $1.001 \mathrm{E}+0$ & $1.073 \mathrm{E}-4$ & $1.004 \mathrm{E}+0$ & $3.287 \mathrm{E}-5$ & $1.002 \mathrm{E}+0$ & $3.287 \mathrm{E}-5$ & $1.002 \mathrm{E}+0$ \\
\hline $1.073 \mathrm{E}-4$ & $1.004 \mathrm{E}+0$ & $3.456 \mathrm{E}-4$ & $1.010 \mathrm{E}+0$ & $1.073 \mathrm{E}-4$ & $1.004 \mathrm{E}+0$ & $1.073 \mathrm{E}-4$ & $1.004 \mathrm{E}+0$ \\
\hline $3.456 \mathrm{E}-4$ & $1.010 \mathrm{E}+0$ & $1.121 \mathrm{E}-3$ & $1.022 \mathrm{E}+0$ & $3.456 \mathrm{E}-4$ & $1.010 \mathrm{E}+0$ & $3.456 \mathrm{E}-4$ & $1.008 \mathrm{E}+0$ \\
\hline $1.121 \mathrm{E}-3$ & $1.022 \mathrm{E}+0$ & $3.682 \mathrm{E}-3$ & $1.046 \mathrm{E}+0$ & $1.121 \mathrm{E}-3$ & $1.020 \mathrm{E}+0$ & $1.121 \mathrm{E}-3$ & $1.016 \mathrm{E}+0$ \\
\hline $3.682 \mathrm{E}-3$ & $1.047 \mathrm{E}+0$ & $1.000 \mathrm{E}-2$ & $1.076 \mathrm{E}+0$ & $3.682 \mathrm{E}-3$ & $1.039 \mathrm{E}+0$ & $3.682 \mathrm{E}-3$ & $1.031 \mathrm{E}+0$ \\
\hline $1.000 \mathrm{E}-2$ & $1.085 \mathrm{E}+0$ & $2.500 \mathrm{E}-2$ & $1.110 \mathrm{E}+0$ & $1.000 \mathrm{E}-2$ & $1.063 \mathrm{E}+0$ & $1.000 \mathrm{E}-2$ & $1.052 \mathrm{E}+0$ \\
\hline $2.500 \mathrm{E}-2$ & $1.132 \mathrm{E}+0$ & $5.000 \mathrm{E}-2$ & $1.135 \mathrm{E}+0$ & $2.500 \mathrm{E}-2$ & $1.091 \mathrm{E}+0$ & $2.500 \mathrm{E}-2$ & $1.079 \mathrm{E}+0$ \\
\hline $5.000 \mathrm{E}-2$ & $1.169 \mathrm{E}+0$ & $7.000 \mathrm{E}-2$ & $1.142 \mathrm{E}+0$ & $5.000 \mathrm{E}-2$ & $1.112 \mathrm{E}+0$ & $5.000 \mathrm{E}-2$ & $1.098 \mathrm{E}+0$ \\
\hline $7.000 \mathrm{E}-2$ & $1.181 \mathrm{E}+0$ & $1.000 \mathrm{E}-1$ & $1.139 \mathrm{E}+0$ & $7.000 \mathrm{E}-2$ & $1.117 \mathrm{E}+0$ & $7.000 \mathrm{E}-2$ & $1.103 \mathrm{E}+0$ \\
\hline $1.000 \mathrm{E}-1$ & $1.183 \mathrm{E}+0$ & $1.500 \mathrm{E}-1$ & $1.117 \mathrm{E}+0$ & $1.000 \mathrm{E}-1$ & $1.113 \mathrm{E}+0$ & $1.000 \mathrm{E}-1$ & $1.099 \mathrm{E}+0$ \\
\hline $1.500 \mathrm{E}-1$ & $1.165 \mathrm{E}+0$ & $2.000 \mathrm{E}-1$ & $1.085 \mathrm{E}+0$ & $1.500 \mathrm{E}-1$ & $1.090 \mathrm{E}+0$ & $1.500 \mathrm{E}-1$ & $1.076 \mathrm{E}+0$ \\
\hline $2.000 \mathrm{E}-1$ & $1.134 \mathrm{E}+0$ & $2.250 \mathrm{E}-1$ & $1.067 \mathrm{E}+0$ & $2.000 \mathrm{E}-1$ & $1.058 \mathrm{E}+0$ & $2.000 \mathrm{E}-1$ & $1.044 \mathrm{E}+0$ \\
\hline $2.250 \mathrm{E}-1$ & $1.116 \mathrm{E}+0$ & $2.500 \mathrm{E}-1$ & $1.050 \mathrm{E}+0$ & $2.250 \mathrm{E}-1$ & $1.041 \mathrm{E}+0$ & $2.250 \mathrm{E}-1$ & $1.027 \mathrm{E}+0$ \\
\hline $2.500 \mathrm{E}-1$ & $1.099 \mathrm{E}+0$ & $3.000 \mathrm{E}-1$ & $1.015 \mathrm{E}+0$ & $2.500 \mathrm{E}-1$ & $1.024 \mathrm{E}+0$ & $2.500 \mathrm{E}-1$ & $1.010 \mathrm{E}+0$ \\
\hline $3.000 \mathrm{E}-1$ & $1.063 \mathrm{E}+0$ & $3.500 \mathrm{E}-1$ & $9.813 \mathrm{E}-1$ & $3.000 \mathrm{E}-1$ & $9.894 \mathrm{E}-1$ & $3.000 \mathrm{E}-1$ & $9.759 \mathrm{E}-1$ \\
\hline $3.500 \mathrm{E}-1$ & $1.029 \mathrm{E}+0$ & $4.000 \mathrm{E}-1$ & $9.498 \mathrm{E}-1$ & $3.500 \mathrm{E}-1$ & $9.565 \mathrm{E}-1$ & $3.500 \mathrm{E}-1$ & $9.435 \mathrm{E}-1$ \\
\hline $4.000 \mathrm{E}-1$ & $9.961 \mathrm{E}-1$ & $4.500 \mathrm{E}-1$ & $9.204 \mathrm{E}-1$ & $4.000 \mathrm{E}-1$ & $9.257 \mathrm{E}-1$ & $4.000 \mathrm{E}-1$ & $9.130 \mathrm{E}-1$ \\
\hline $4.500 \mathrm{E}-1$ & $9.657 \mathrm{E}-1$ & $5.000 \mathrm{E}-1$ & $8.931 \mathrm{E}-1$ & $4.500 \mathrm{E}-1$ & $8.970 \mathrm{E}-1$ & $4.500 \mathrm{E}-1$ & $8.847 \mathrm{E}-1$ \\
\hline $5.000 \mathrm{E}-1$ & $9.374 \mathrm{E}-1$ & $5.500 \mathrm{E}-1$ & $8.678 \mathrm{E}-1$ & $5.000 \mathrm{E}-1$ & $8.703 \mathrm{E}-1$ & $5.000 \mathrm{E}-1$ & $8.583 \mathrm{E}-1$ \\
\hline $5.500 \mathrm{E}-1$ & $9.111 \mathrm{E}-1$ & $6.000 \mathrm{E}-1$ & $8.443 \mathrm{E}-1$ & $5.500 \mathrm{E}-1$ & $8.456 \mathrm{E}-1$ & $5.500 \mathrm{E}-1$ & $8.339 \mathrm{E}-1$ \\
\hline $6.000 \mathrm{E}-1$ & $8.866 \mathrm{E}-1$ & $6.500 \mathrm{E}-1$ & $8.224 \mathrm{E}-1$ & $6.000 \mathrm{E}-1$ & $8.226 \mathrm{E}-1$ & $6.000 \mathrm{E}-1$ & $8.113 \mathrm{E}-1$ \\
\hline $6.500 \mathrm{E}-1$ & $8.639 \mathrm{E}-1$ & $7.000 \mathrm{E}-1$ & $8.020 \mathrm{E}-1$ & $6.500 \mathrm{E}-1$ & $8.013 \mathrm{E}-1$ & $6.500 \mathrm{E}-1$ & $7.902 \mathrm{E}-1$ \\
\hline $7.000 \mathrm{E}-1$ & $8.426 \mathrm{E}-1$ & $7.500 \mathrm{E}-1$ & $7.830 \mathrm{E}-1$ & $7.000 \mathrm{E}-1$ & $7.814 \mathrm{E}-1$ & $7.000 \mathrm{E}-1$ & $7.706 \mathrm{E}-1$ \\
\hline $7.500 \mathrm{E}-1$ & $8.227 \mathrm{E}-1$ & $8.000 \mathrm{E}-1$ & $7.652 \mathrm{E}-1$ & $7.500 \mathrm{E}-1$ & $7.628 \mathrm{E}-1$ & $7.500 \mathrm{E}-1$ & $7.523 \mathrm{E}-1$ \\
\hline $8.000 \mathrm{E}-1$ & $8.041 \mathrm{E}-1$ & $8.500 \mathrm{E}-1$ & $7.485 \mathrm{E}-1$ & $8.000 \mathrm{E}-1$ & $7.455 \mathrm{E}-1$ & $8.000 \mathrm{E}-1$ & $7.352 \mathrm{E}-1$ \\
\hline $8.500 \mathrm{E}-1$ & $7.867 \mathrm{E}-1$ & $9.000 \mathrm{E}-1$ & $7.328 \mathrm{E}-1$ & $8.500 \mathrm{E}-1$ & $7.292 \mathrm{E}-1$ & $8.500 \mathrm{E}-1$ & $7.191 \mathrm{E}-1$ \\
\hline $9.000 \mathrm{E}-1$ & $7.702 \mathrm{E}-1$ & $9.500 \mathrm{E}-1$ & $7.180 \mathrm{E}-1$ & $9.000 \mathrm{E}-1$ & $7.139 \mathrm{E}-1$ & $9.000 \mathrm{E}-1$ & $7.040 \mathrm{E}-1$ \\
\hline $9.500 \mathrm{E}-1$ & $7.548 \mathrm{E}-1$ & $9.750 \mathrm{E}-1$ & $7.110 \mathrm{E}-1$ & $9.500 \mathrm{E}-1$ & $6.995 \mathrm{E}-1$ & $9.500 \mathrm{E}-1$ & $6.898 \mathrm{E}-1$ \\
\hline $9.700 \mathrm{E}-1$ & $7.488 \mathrm{E}-1$ & $9.800 \mathrm{E}-1$ & $7.096 \mathrm{E}-1$ & $9.750 \mathrm{E}-1$ & $6.926 \mathrm{E}-1$ & $9.750 \mathrm{E}-1$ & $6.829 \mathrm{E}-1$ \\
\hline $9.800 \mathrm{E}-1$ & $7.459 \mathrm{E}-1$ & $9.825 \mathrm{E}-1$ & $7.089 \mathrm{E}-1$ & $9.800 \mathrm{E}-1$ & $6.912 \mathrm{E}-1$ & $9.800 \mathrm{E}-1$ & $6.817 \mathrm{E}-1$ \\
\hline \multirow[t]{2}{*}{$9.850 \mathrm{E}-1$} & $7.459 \mathrm{E}-1$ & $9.830 \mathrm{E}-1$ & $7.088 \mathrm{E}-1$ & $9.830 \mathrm{E}-1$ & $6.904 \mathrm{E}-1$ & $9.850 \mathrm{E}-1$ & $6.817 \mathrm{E}-1$ \\
\hline & & $9.850 \mathrm{E}-1$ & $7.088 \mathrm{E}-1$ & $9.850 \mathrm{E}-1$ & $6.904 \mathrm{E}-1$ & & \\
\hline
\end{tabular}

*same values as in Table A2 are given here for convenience. 
Table A5. The dimensional and dimensionless quantities and scaling factors.

\begin{tabular}{|c|c|c|}
\hline Dimensional quantity & Dimensionless quantity & Remark \\
\hline$\lambda$ & $\lambda / \xi k=1$ & \\
\hline$\xi$ & $\frac{\xi}{\xi k}=\frac{1}{k}$ & \\
\hline$r$ & $\frac{r}{\xi k}$ & \\
\hline$\pi R^{2}$ & $\Phi_{0} / \bar{b}_{k}=2 \pi / k \bar{b}_{k}$ & \\
\hline$|\psi|$ & $\frac{|\psi|}{\sqrt{\alpha / \beta}}$ & \\
\hline$f(r)$ & $\frac{f(r)}{f_{\infty}}$ & \\
\hline$\vartheta$ & $\vartheta$ & $2 \pi$ \\
\hline$a_{\varphi}(r)$ & $\frac{a_{\varphi}(r)}{k \xi B_{c} \sqrt{2}}=\frac{a_{\varphi}(r)}{\xi B_{c 2}}$ & $\xi B_{c 2}=\frac{\Phi_{0}}{2 \pi \xi}$ \\
\hline$B$ & $\frac{H}{k H_{c} \sqrt{2}}=\frac{H}{H_{c 2}}$ & $B=\mu_{0} H$ \\
\hline$B_{c 1}$ & $\frac{H_{c 1}}{H_{c} \sqrt{2}}$ & $B_{c}=\frac{k_{12} B_{c 2}}{k}$ \\
\hline$B_{c 2}$ & $\frac{B_{c 2}}{B_{c} \sqrt{2}}=k$ & $B_{c 2}=\mu_{0} H_{c 2}$ \\
\hline$B_{c}$ & $\frac{B_{c}}{B_{c} \sqrt{2}}=\frac{1}{\sqrt{2}}$ & $k_{12}=\frac{1}{\sqrt{2}}$ \\
\hline$j_{\varphi}(r)$ & $\frac{j_{\varphi}(r) \xi k}{H_{c} \sqrt{2}}$ & \\
\hline$q_{\varphi}(r)$ & $\frac{q_{\varphi}(r)}{k \xi B_{c} \sqrt{2}}=\frac{q_{\varphi}(r)}{\xi B_{c 2}}$ & $\frac{B_{c 2}}{B_{c} \sqrt{2}}=k$ \\
\hline$\Phi_{0}$ & $\frac{\Phi_{0}}{\lambda^{2} \mu_{0} H_{c} \sqrt{2}}=\frac{2 \pi}{\mu_{0} k}$ & $B_{c 2}=\frac{\Phi_{0}}{2 \pi \xi^{2}}$ \\
\hline$F$ & $\frac{F}{\mu_{0} H_{c}^{2}}$ & $\frac{F}{k^{2} \mu_{0} H_{c}^{2}}=\frac{2 F}{\mu_{0} H_{c 2}^{2}}$ \\
\hline$M_{0}$ & $\frac{M_{0}}{B_{c} \sqrt{2}}$ and $\frac{M_{0}}{B_{c 2}}$ & \\
\hline
\end{tabular}


Submit or recommend next manuscript to SCIRP and we will provide best service for you:

Accepting pre-submission inquiries through Email, Facebook, LinkedIn, Twitter, etc. A wide selection of journals (inclusive of 9 subjects, more than 200 journals)

Providing 24-hour high-quality service

User-friendly online submission system

Fair and swift peer-review system

Efficient typesetting and proofreading procedure

Display of the result of downloads and visits, as well as the number of cited articles Maximum dissemination of your research work

Submit your manuscript at: http://papersubmission.scirp.org/

Or contact jmp@scirp.org 